Kametaka, Y.

Osaka J. Math.

13 (1976), 11-66

\title{
ON THE NONLINEAR DIFFUSION EQUATION OF KOLMOGOROV-PETROVSKII-PISKUNOV TYPE
}

\author{
YosHINORI KAMETAKA
}

(Received December 5, 1974)

\section{Contents}

Introduction .......................................................... 11

Part I. Travelling waves

1. Existence, uniqueness and properties of the travelling wave $\ldots \ldots \ldots \ldots \ldots . \ldots 17$

2. KPP transform of the travelling wave ..................................... 24

3. Second existence theorem for the travelling wave ......................... 27

Part II. Asymptotic behavior of the time dependent solution

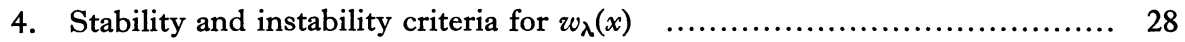

5. A problem with higher space dimension ............................... 38

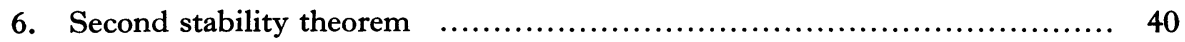

Part III. Method of KPP transform

7. Fundamental theorem of KPP .......................................... 41

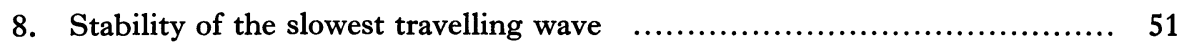

9. Stability of the travelling wave with arbitrary speed ..................... 57

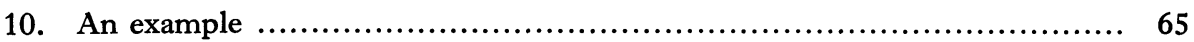

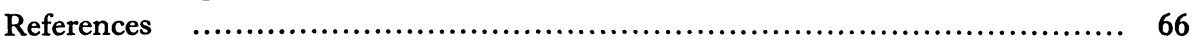

\section{Introduction}

Kolmogorov, Petrovskii and Piskunov [1], whom we shall refer to as KPP, studied the initial value problem for a semilinear diffusion equation

$$
\begin{aligned}
& \left\{\begin{array}{l}
L u=f(u), \quad 0 \leqslant u \leqslant 1 \\
u(x, 0)=u_{0}(x) \quad x \in R^{1},
\end{array} \quad(x, t) \in R^{1} \times(0, \infty),\right. \\
& \left(\text { where } L=\frac{\partial}{\partial t}-\left(\frac{\partial}{\partial x}\right)^{2}, \quad u=u(x, t),\right)
\end{aligned}
$$

under the conditions:
(i)
$f(\xi) \in C^{\infty}[0,1]$
(ii)
$f(0)=f(1)=0, \quad f^{\prime}(0)>0>f^{\prime}(1)$,
(iii)
$f(\xi)>0, \quad f_{0}(\xi) \equiv f^{\prime}(0) \xi-f(\xi) \geqslant 0 \quad \xi \in(0,1)$. 
These conditions are supposed to be satisfied throughout this paper. Replacing $u$ by $w(x+2 \lambda t)$ the equation (1) is reduced to

$$
\begin{aligned}
& w^{\prime \prime}-2 \lambda w^{\prime}+f(w)=0, \quad 0 \leqslant w \leqslant 1 \quad x \in R^{1}, \\
& \text { (where }{ }^{\prime}=\frac{d}{d x}, \quad w=w(x) . \text { ) }
\end{aligned}
$$

Since the equation (2) is invariant under the transformation $(x, \lambda) \rightarrow(-x,-\lambda)$, we shall treat the case of $\lambda \geqslant 0$.

KPP showed that

$$
\lambda_{0}=\sqrt{f^{\prime}(0)}
$$

is the critical value in the following sense:

If $0 \leqslant \lambda<\lambda_{0}$ then (2) has only trivial solutions $w \equiv 0$ and $w \equiv 1$. (As it is easy to prove this fact, we omit it.)

If $\lambda \geqslant \lambda_{0}$ then (2) andthe normalizing condition $w(0)=1 / 2$ determine the unique solution $w_{\lambda}(x)$. It satisfies automatically $w_{\lambda}^{\prime}(x)>0 x \in R^{1}, w_{\lambda}(-\infty)=0$ and $w_{\lambda}(+\infty)=1$. (This fact is proved in Part I for the sake of completeness.)

We call $w_{\lambda}(x)$ or $w_{\lambda}(x+2 \lambda t)$ the travelling wave of $(1)$ with speed $-2 \lambda$.

In Part I we investigate the equation (2) in case of $\lambda \geqslant \lambda_{0}$. The existence and uniqueness of the nontrivial solution $w_{\lambda}(x)$ will be established. We shall give also the detailed properties of $w_{\lambda}(x)$ which play important roles in Parts II and III. We also investigate

$$
w^{\prime \prime}-\left\{2 \lambda+g^{\prime}(w)\right\} w^{\prime}+f(w)=0, \quad 0 \leqslant w \leqslant 1 \quad x \in R^{1}
$$

under suitable conditions on $g$. The existence and uniqueness of solution of (4) will be needed in Part III. The nontrivial solution $w(x)$ of $(4)$ is also called the travelling wave for the sake of convenience.

Replacing $u(x+2 \lambda t, t)$ by $u(x, t)$ the problem (1) is reduced to

$$
\left\{\begin{array}{l}
{\left[L+2 \lambda \frac{\partial}{\partial x}\right] u=f(u), \quad 0 \leqslant u \leqslant 1 \quad(x, t) \in R^{1} \times(0, \infty),} \\
u(x, 0)=u_{0}(x) \quad x \in R^{1} .
\end{array}\right.
$$

In Part II we propose two kinds of criteria on the initial function $u_{0}(x)$ which conclude

$$
\lim _{t \rightarrow \infty} \sup _{x \leqslant A} u(x, t)=0 \quad \text { for any } A
$$

or

$$
\lim _{t \rightarrow \infty} \sup _{x \in R^{1}}\left|u(x, t)-w_{\lambda}(x+c)\right|=0 \quad \text { for some } c .
$$

An application of this result to a problem with higher space dimension is considered also. Adding the assumption: 


$$
f^{\prime}(\xi) \leqslant f^{\prime}(0) \quad \xi \in(0,1),
$$

we propose certain criterion on the initial function $u_{0}(x)$ which concludes

$$
\lim _{t \rightarrow \infty} \sup _{x \in R^{1}}\left|u(x, t)-w_{\lambda}(x)\right|=0 .
$$

KPP obtained in [1] a remarkable result: If the solution $u(x, t)$ of $(1)$ has the initial function $u_{0}(x)=1(x>0),=0(x<0)$, then we have

$$
(\operatorname{sgn} x)\left\{u\left(x+u^{-1}(\xi, \tau), \tau\right)-w_{\lambda_{0}}\left(x+w_{\lambda_{0}}{ }^{-1}(\xi)\right)\right\} \searrow 0
$$

as $\tau \nearrow+\infty$, uniformly with respect to $x \in R^{1}$ and uniformly with respect to $\xi$ on every closed subinterval of $(0,1)$.

$$
\lim _{\tau \rightarrow \infty} \frac{\partial}{\partial \tau} u^{-1}(\xi, \tau)=-2 \lambda_{0} .
$$

Here $u^{-1}(\xi, \tau)$ is given by the implicit relation $u\left(u^{-1}(\xi, \tau), \tau\right)=\xi$ for $(\xi, \tau) \in(0,1)$ $\times(0, \infty)$. This is well defined since we have $\frac{\partial}{\partial x} u(x, t)>0$ for $(x, t) \in R^{1} \times$ $(0, \infty)$. Their proof is based on the fascinating method which we would like to call the method of KPP transform. Part III is devoted to showing the effectiveness of the method of KPP transform. Let us introduce the class $M$ of smooth functions by

$$
M=\left\{u(x) ; \quad u^{\prime}(x)>0 \quad x \in R^{1}, \quad u(-\infty)=0, \quad u(+\infty)=1\right\} .
$$

Suppose that the smooth function $u(x, t)$ belongs to the class $M$ for any $t \geqslant 0$. Then we can define the smooth function $u^{-1}(\xi, \tau)$ by the implicit relation

$$
u\left(u^{-1}(\xi, \tau), \tau\right)=\xi \quad(\xi, \tau) \in(0,1) \times[0, \infty) .
$$

Let us call the new function $\mathfrak{a}(\xi, \tau)$ given by

$$
\mathfrak{a}(\xi, \tau)=u^{\prime}\left(u^{-1}(\xi, \tau), \tau\right) \quad(\xi, \tau) \in(0,1) \times[0, \infty)
$$

the KPP transform of $u(x, t)$. Here and hereafter for any smooth function $f$ of two independent variables $(x, t)$ or $(\xi, \tau) f^{\prime}$ and $\dot{f}$ mean the partial differentiations with respect to the first and the second variable respectively. If the above $u(x, t)=u(x)$ is independent of $t$, then $u^{-1}(\xi, \tau)=u^{-1}(\xi)$ and $\mathfrak{a}(\xi, \tau)=\mathfrak{a}(\xi)$ are also independent of $\tau$. The transformation

$$
\left\{\begin{array} { l } 
{ \xi = u ( x , t ) , } \\
{ \tau = t }
\end{array} \text { or } \quad \left\{\begin{array}{l}
x=u^{-1}(\xi, \tau), \\
t=\tau
\end{array}\right.\right.
$$

gives diffeomorphism between the region $R^{1} \times[0, \infty)$ of the $(x, t)$-plane and the region $(0,1) \times[0, \infty)$ of the $(\xi, \tau)$-plane. Hereafter, let $u(x, t)$ be the solution of 
(1) with $u_{0}(x) \in M$. It follows easily that $u(x, t) \in M$ for any $t \geqslant 0$. Differentiating (8) we have

$$
\left\{\begin{array}{l}
\left(u^{-1}\right)^{\prime}(\xi, \tau)=\frac{1}{u^{\prime}(x, t)}=\frac{1}{\hat{u}(\xi, \tau)}, \\
\left(u^{-1}\right)^{\cdot}(\xi, \tau)=-\frac{\dot{u}(x, t)}{u^{\prime}(x, t)}=-\left\{\hat{u}^{\prime}(\xi, \tau)+\frac{f(\xi)}{\hat{u}(\xi, \tau)}\right\} .
\end{array}\right.
$$

The transformation (10) induces the rules of transformations between the partial differentiations $\left(\frac{\partial}{\partial \xi}, \frac{\partial}{\partial \tau}\right)$ and $\left(\frac{\partial}{\partial x}, \frac{\partial}{\partial t}\right)$ :

$$
\begin{aligned}
& \left\{\begin{array}{l}
\frac{\partial}{\partial \xi}=\frac{1}{u^{\prime}(x, t)} \frac{\partial}{\partial x}, \\
\frac{\partial}{\partial \tau}=\frac{\partial}{\partial t}-\frac{\dot{u}(x, t)}{u^{\prime}(x, t)} \frac{\partial}{\partial x},
\end{array}\right. \\
& \left\{\begin{array}{l}
\frac{\partial}{\partial x}=\hat{u}(\xi, \tau) \frac{\partial}{\partial \xi}, \\
\frac{\partial}{\partial t}=\frac{\partial}{\partial \tau}+\left\{\hat{u}(\xi, \tau) \hat{u}^{\prime}(\xi, \tau)+f(\xi)\right\} \frac{\partial}{\partial \xi} .
\end{array}\right.
\end{aligned}
$$

Especially we have

$$
L=\frac{\partial}{\partial t}-\left(\frac{\partial}{\partial x}\right)^{2}=\frac{\partial}{\partial \tau}-\hat{u}^{2}(\xi, \tau)\left(\frac{\partial}{\partial \xi}\right)^{2}+f(\xi) \frac{\partial}{\partial \xi}=\hat{L}_{\hat{u}} .
$$

It follows from (12) that

$$
\begin{aligned}
\hat{u}^{\prime}(\xi, \tau) & =\frac{u^{\prime \prime}(x, t)}{u^{\prime}(x, t)} \\
\hat{u}^{\prime \prime}(\xi, \tau) & =\frac{1}{u^{\prime}(x, t)}\left\{\frac{u^{\prime \prime}(x, t)}{u^{\prime}(x, t)}\right\}^{\prime} \\
& =\frac{1}{u^{\prime}(x, t)}\left\{\frac{u^{\prime \prime \prime}(x, t)}{u^{\prime}(x, t)}-\left(\frac{u^{\prime \prime}(x, t)}{u^{\prime}(x, t)}\right)^{2}\right\}
\end{aligned}
$$

Differentiating (1) we have

$$
\left[L-f^{\prime}(u)\right] u^{\prime}=0
$$

This can be rewritten as

$$
\left[\hat{L}_{\hat{u}}-f^{\prime}(\xi)\right] \hat{u}=0
$$

or

$$
\grave{u}(\xi, \tau)=\hat{u}^{2}(\xi, \tau) \hat{u}^{\prime \prime}(\xi, \tau)-f(\xi) \hat{u}^{\prime}(\xi, \tau)+f^{\prime}(\xi) \hat{u}(\xi, \tau)
$$




$$
=u^{\prime \prime \prime}(x, t)-\frac{\left(u^{\prime \prime}(x, t)\right)^{2}}{u^{\prime}(x, t)}-f(u(x, t)) \frac{u^{\prime \prime}(x, t)}{u^{\prime}(x, t)}+f^{\prime}(u(x, t)) u^{\prime}(x, t) .
$$

Since we have

$$
w_{\lambda}^{\prime \prime}(x)-2 \lambda w_{\lambda}^{\prime}(x)+f\left(w_{\lambda}(x)\right)=0
$$

it follows

$$
\hat{w}_{\lambda}{ }^{\prime}(\xi)+\frac{f(\xi)}{\hat{w}_{\lambda}(\xi)}=2 \lambda
$$

Differentiating (21) with respect to $\xi$ we get

$$
0=\hat{w}_{\lambda}^{2}(\xi) \hat{w}_{\lambda}^{\prime \prime}(\xi)-f(\xi) \hat{w}_{\lambda}^{\prime}(\xi)+f^{\prime}(\xi) \hat{w}_{\lambda}(\xi) .
$$

Subtracting (22) from (19) we have

$$
\left[\hat{L}_{\hat{u}}-\left\{f^{\prime}(\xi)+\left(\hat{u}+\hat{w}_{\lambda}\right) \hat{w}_{\lambda}{ }^{\prime \prime}\right\}\right]\left(\hat{u}-\hat{w}_{\lambda}\right)=0 .
$$

Putting

$$
\begin{aligned}
c_{1}(x, t) & =\left.\left\{f^{\prime}(\xi)+\left(\hat{u}+\hat{w}_{\lambda}\right) \hat{w}_{\lambda}{ }^{\prime \prime}\right\}\right|_{\xi=u(x, t), \tau=t} \\
& =f^{\prime}(u(x, t))+\left\{u^{\prime}(x, t)+\hat{w}_{\lambda}(u(x, t))\right\} \hat{w}_{\lambda}^{\prime \prime}(u(x, t)),
\end{aligned}
$$

we can rewrite (23) in the form

$$
\left[L-c_{1}(x, t)\right]\left(u^{\prime}-\hat{w}_{\lambda}(u)\right)=0 .
$$

Differentiating (18) with respect to $\tau$ we get

$$
\left[\hat{L}_{\hat{u}}-\left\{f^{\prime}(\xi)+2 \hat{a} \hat{u}^{\prime \prime}\right\}\right] \dot{\vec{u}}=0 .
$$

Putting

$$
\begin{aligned}
c_{2}(x, t) & =\left.\left\{f^{\prime}(\xi)+2 \hat{u} \hat{u}^{\prime \prime}\right\}\right|_{\xi=u(x, t), \tau=t} \\
& =f^{\prime}(u(x, t))+2\left\{\frac{u^{\prime \prime \prime}(x, t)}{u^{\prime}(x, t)}-\left(\frac{u^{\prime \prime}(x, t)}{u^{\prime}(x, t)}\right)^{2}\right\},
\end{aligned}
$$

we can rewrite (26) in the form

$$
\left[L-c_{2}(x, t)\right] \dot{\hat{u}}(u(x, t), t)=0 .
$$

For any function $h(\xi, \tau)$, subtracting $\left[\hat{L}_{h}-f^{\prime}(\xi)\right] h$ from (18) we have

$$
\left[\hat{L}_{\hat{u}}-\left\{f^{\prime}(\xi)+(\hat{u}+h) h^{\prime \prime}\right\}\right](\hat{u}-h)=-\left[\hat{L}_{h}-f^{\prime}(\xi)\right] h .
$$

Let us call the $(\hat{u}, \xi)$-plane the phase plane. Roughly speaking the main results in Part III are that all the travelling waves are stable from above and below on the phase plane. Especially corresponding to the original results of KPP, the 
slowest travelling wave is stable from above almost in the large on the phase plane. In Part III the following standard comparison theorem plays an essential role.

Comparison Theorem (see [2] Chapter 10).

Suppose that $c(x, t)$ satisfies the growth condition:

$$
c(x, t) \leqslant C\left\{x^{2}+1\right\} \quad(x, t) \in R^{1} \times[0, T]
$$

for some $C \geqslant 0$. Suppose that $u(x, t)$ satisfies the regularity conditions:

$$
\begin{aligned}
& u(x, t) \in C^{0}\left(R^{1} \times[0, T]\right), \\
& u^{\prime}(x, t), u^{\prime \prime}(x, t), \dot{u}(x, t) \in C^{0}\left(R^{1} \times(0, T]\right)
\end{aligned}
$$

and the growth condition:

$$
u(x, t) \geqslant-M e^{K_{0} x^{2}} \quad(x, t) \in R^{1} \times[0, T]
$$

for some $M \geqslant 0$ and $K_{0} \geqslant 0$. Then the differential inequality

$$
\left\{\begin{array}{l}
{[L-c(x, t)] u \geqslant 0 \quad(x, t) \in R^{1} \times(0, T],} \\
u(x, 0) \geqslant 0 \quad x \in R^{1}
\end{array}\right.
$$

implies

$$
u(x, t) \geqslant 0 \quad(x, t) \in R^{1} \times[0, T] .
$$

The following simple fact also plays an important role. If $u_{0}(x) \in \mathscr{B}^{3}\left(R^{1}\right)\left(u_{0}(x)\right.$ has the bounded continuous derivatives up to the third order), then the solution $u(x, t)$ of (1) satisfies

$$
\sup _{x \in R^{1}}\left|\left(\frac{\partial}{\partial x}\right)^{k} u(x, t)\right| \leqslant e^{A t+B} \quad t \geqslant 0, k=0,1,2,3
$$

for some $A \geqslant 0$ and $B \geqslant 0$. This can be shown as follows. Define the approximate solution $u_{j}(x, t)$ by the iteration

$$
\left\{\begin{array}{l}
u_{0}(x, t)=\int_{-\infty}^{\infty} H(x-y, t) u_{0}(y) d y, \\
u_{j}(x, t)=u_{0}(x, t)+\int_{0}^{t} \int_{-\infty}^{\infty} H(x-y, t-s) f\left(u_{j-1}(y, s)\right) d y d s \quad j=1,2,3, \cdots .
\end{array}\right.
$$

Here and hereafter

$$
H(x, t)=\frac{1}{\sqrt{4 \pi t}} e^{-x^{2} / 4 t}
$$

represents the fundamental solution of the diffusion equation. Then it is easy to see that there exist $A \geqslant 0$ and $B \geqslant 0$ such that 


$$
\begin{array}{ll}
\sup _{x \in R^{1}}\left|\left(\frac{\partial}{\partial x}\right)^{k} u_{j}(x, t)\right| \leqslant e^{A t+B} & t \geqslant 0, \quad k=0,1,2,3, \\
& j=0,1,2,3, \cdots .
\end{array}
$$

It is also easy to see that for $k=0,1,2,3$,

$$
\lim _{j \rightarrow \infty} \sup _{R^{1} \times[0, T]}\left|\left(\frac{\partial}{\partial x}\right)^{k}\left\{u_{j}(x, t)-u(x, t)\right\}\right|=0
$$

for any $T>0$. (31) and (32) proves (30).

\section{Part I Travelling Waves}

\section{Existence, uniqueness and properties of the travelling wave}

In this section we study

$$
w^{\prime \prime}-\left\{2 \lambda+g^{\prime}(w)\right\} w^{\prime}+f(w)=0, \quad 0 \leqslant w \leqslant 1 \quad x \in R^{1}
$$

under the condition

$$
g(0)=g^{\prime}(0)=0, \quad g(\xi) \in C^{\infty}[0,1] .
$$

The assumptions on $f$ are stated in Introduction. Put

$$
2 \mu=2 \lambda+g^{\prime}(1)
$$

Then (4) is equivalent to

$$
\left(\begin{array}{c}
w-1 \\
w^{\prime}
\end{array}\right)^{\prime}=\left(\begin{array}{cc}
0 & 1 \\
-f^{\prime}(1) & 2 \mu
\end{array}\right)\left(\begin{array}{c}
w-1 \\
w^{\prime}
\end{array}\right)+\cdots
$$

Here "..." represents the higher order term for small $(w-1, w)$. The coefficient matrix in (1.3) has two distinct eigenvalues

$$
\tau_{ \pm}=\tau_{ \pm}(\mu)=\mu \pm \sqrt{\mu^{2}-f^{\prime}(1)}
$$

with opposite sign $\left(\tau_{+}>0>\tau_{-}\right)$. The singular point $\left(w-1, w^{\prime}\right)=(0,0)$ is the saddle. Well known theory of 2-dimensional autonomous system gives

Lemma 1.1 (see [3] Chapter 13). There exists a $C^{\infty}$ function $w(x)$ defined and satisfying (4) in the interval $[0, \infty)$. Moreover it satisfies

$$
\begin{gathered}
0<w(x)<1, \quad w^{\prime}(x)>0 \quad x \geqslant 0, \\
\left\{\begin{array}{cll}
1-w(x)=a_{1} e^{\tau_{-} x}\left\{1+O\left(e^{-\delta x}\right)\right\} & \text { as } \quad x \rightarrow+\infty, \\
-w^{\prime}(x)=\tau_{-} a_{1} e^{\tau_{-} x}\left\{1+O\left(e^{-\delta x}\right)\right\} & \text { as } \quad x \rightarrow+\infty
\end{array}\right.
\end{gathered}
$$

for some $a_{1}>0$ and $\delta>0$, 


$$
\hat{w}(\xi)=w^{\prime}\left(w^{-1}(\xi)\right) \in C^{\infty}[w(0), 1],
$$

$\left(\right.$ where $w^{-1}(\xi)$ is given by $w\left(w^{-1}(\xi)\right)=\xi$ for $\left.\xi \in[w(0), 1],\right)$ and

$$
\hat{w}(\xi)>0 \quad \xi \in[w(0), 1], \quad \hat{w}(1)=0, \quad \hat{w}^{\prime}(1)=\tau_{-} .
$$

The above $w(x)$ is unique in the following sense.

Theorem 1.1. We assume $\lambda \geqslant 0$. Suppose that $v(x) \in C^{2}(0, \infty)$ satisfies

$$
v^{\prime \prime}-\left\{2 \lambda+g^{\prime}(v)\right\} v^{\prime}+f(v)=0, \quad 0 \leqslant v \leqslant 1 \quad x>0 .
$$

If $v(x)$ is not identically equal to 0 or 1 , then we have

$$
v(x)=w(x+c) \quad x>x_{0}
$$

for some $c$ and $x_{0}$. Here $w(x)$ is the function stated in Lemma 1.1.

Proof. Integrating (1.9) on the interval $[0, x]$, we have

$$
v^{\prime}(x)=O(x) \quad \text { as } \quad x \rightarrow+\infty .
$$

For any $\varepsilon>0$, we can rewrite (1.9) in the form

$$
\left(\frac{d}{d x}-\varepsilon\right)\left\{v^{\prime}-(2 \lambda-\varepsilon) v-g(v)\right\}=h(v) .
$$

Here

$$
h(v)=\varepsilon(2 \lambda-\varepsilon) v-f(v)+\varepsilon g(v) .
$$

Integrating (1.12) and using (1.11), we get

$$
\begin{aligned}
& v^{\prime}(x)-(2 \lambda-\varepsilon) v(x)-g(v(x)) \\
= & -\int_{x}^{\infty} e^{\varepsilon(x-y)} h(v(y)) d y=-\int_{0}^{\infty} e^{-\varepsilon y} h(v(x+y)) d y .
\end{aligned}
$$

Suppose that $v(x)$ is not identically equal to 0 or 1 . Then it is easy to see

$$
v^{\prime}(x)>0 \quad x>x_{1}
$$

or

$$
v^{\prime}(x)<0 \quad x>x_{1}
$$

for some $x_{1} \geqslant 0$. In any case there exists the limit $v(+\infty)=\lim _{x \rightarrow+\infty} v(x)$. By Lebesgue's theorem we have

$$
\lim _{x \rightarrow+\infty} \int_{0}^{\infty} e^{-\varepsilon y} h(v(x+y)) d y=\frac{1}{\varepsilon} h(v(+\infty)) .
$$


(1.14) and (1.17) assure the existence of the limit $v^{\prime}(+\infty)=\lim _{x \rightarrow+\infty} v^{\prime}(x)$ and give

$$
v^{\prime}(+\infty)=\frac{1}{\varepsilon} f(v(+\infty))
$$

This implies

$$
v(+\infty)=1, \quad v^{\prime}(+\infty)=0
$$

or

$$
v(+\infty)=v^{\prime}(+\infty)=0 .
$$

The singular point $\left(v, v^{\prime}\right)=(0,0)$ has the characteristic roots $\sigma_{ \pm}(\lambda)=\lambda \pm \sqrt{\lambda^{2}-\lambda_{0}^{2}}$. Since real parts of $\sigma_{ \pm}(\lambda)$ are non negative for $\lambda \geqslant 0$. This means that this singular point is unstable node, center or spiral. So it does not occur (1.20) and (1.16). It is well known that (1.19) implies (1.10). This proves Theorem 1.1.

Now we proceed to prove the existence of the solution $w(x)$ of (4). Since $f$ and $g$ may be considered as $C^{\infty}$ functions with compact supports defined on the whole real line, then $w(x)$ in Lemma 1.1 can be continued uniquely on the whole real line as a solution of the equation (4). The remaining task is to show

$$
0 \leqslant w(x) \leqslant 1 \quad x \in R^{1}
$$

under suitable assumption on $g$. Put

$$
\sigma_{ \pm}=\sigma_{ \pm}(\lambda)=\lambda \pm \sqrt{\lambda^{2}-\lambda_{0}^{2}} .
$$

Hereafter we assume $\lambda \geqslant \lambda_{0}$. Then we have

$$
\sigma_{+}(\lambda) \geqslant \sigma_{-}(\lambda)>0 \text {. }
$$

We can rewrite (4) in the form

$$
\left(\frac{d}{d x}-\sigma_{ \pm}\right)\left\{\left(\frac{d}{d x}-\sigma_{\mp}\right) w-g(w)\right\}=f_{0}(w)+\sigma_{ \pm} g(w) .
$$

Let us introduce following notations:

(i) In case of $\lambda>\lambda_{0}$

$$
\begin{gathered}
A=\left(\begin{array}{cc}
\sigma_{+}(\lambda) & 0 \\
0 & \sigma_{-}(\lambda)
\end{array}\right), \\
u={ }^{t}\left(u_{+}, u_{-}\right), \quad u_{ \pm}=w^{\prime}-\sigma_{\mp}(\lambda) w-g(w), \\
h(\xi)={ }^{t}\left(h_{+}(\xi), h_{-}(\xi)\right), \quad h_{ \pm}(\xi)=f_{0}(\xi)+\sigma_{ \pm}(\lambda) g(\xi) .
\end{gathered}
$$

(ii) In case of $\lambda=\lambda_{0}$

$$
A=\left(\begin{array}{cc}
\lambda_{0} & 1 \\
0 & \lambda_{0}
\end{array}\right),
$$




$$
\begin{aligned}
& u={ }^{t}\left(u_{+}, u_{-}\right), \quad u_{+}=w, \quad u_{-}=w^{\prime}-\lambda_{0} w-g(w), \\
& h(\xi)={ }^{t}\left(h_{+}(\xi), h_{-}(\xi)\right), \quad h_{+}(\xi)=g(\xi), \quad h_{-}(\xi)=f_{0}(\xi)+\lambda_{0} g(\xi) .
\end{aligned}
$$

Then the equation (4) is equivalent to

$$
\frac{d}{d x} u=A u+h(w(x)) \text {. }
$$

Lemma 1.2. If

$$
h_{-}(\xi)=f_{0}(\xi)+\sigma_{-}(\lambda) g(\xi) \geqslant 0 \quad \xi \in[0,1],
$$

then $w(x)$ in Lemma 1.1 satisfies

$$
0<w(x)<1, \quad w^{\prime}(x)>0 \quad x \in R^{1}
$$

and

$$
\lim _{x \rightarrow-\infty} \sup \frac{1}{|x|} \log \left\{|w(x)|+\left|w^{\prime}(x)\right|\right\} \leqslant-\sigma_{-}(\lambda)<0 .
$$

Proof. The second component of the equation (1.31) and (1.6) yield

$$
\begin{aligned}
u_{-}(x) & =-\int_{x}^{\infty} e^{\sigma_{-}(x-y)} h_{-}(w(y)) d y \\
& =-\int_{0}^{\infty} e^{-\sigma_{-} y} h_{-}(w(x+y)) d y .
\end{aligned}
$$

Suppose that (1.33) is violated. Since $w^{\prime}(x)>0$ on every interval $\left(x_{0}, \infty\right)$ on which $0<w(x)<1$, then we have only the possibility of the following situation. There exists $x_{0}$ such that

$$
w\left(x_{0}\right)=0, \quad w^{\prime}(y)>0 \quad y \geqslant x_{0} .
$$

By (1.32), (1.35) and (1.36) we have

$$
w^{\prime}\left(x_{0}\right)=-\int_{x_{0}}^{\infty} e^{\sigma_{-}\left(x_{0}-y\right)} h_{-}(w(y)) d y \leqslant 0 .
$$

On the other hand by (1.36) we have $w^{\prime}\left(x_{0}\right)>0$. This is a contradiction. This proves (1.33). By (1.33) we have the limit $w(-\infty)=\lim _{x \rightarrow-\infty} w(x)$. By Lebesgue's theorem we have

$$
\lim _{x \rightarrow-\infty} \int_{0}^{\infty} e^{-\sigma_{-} y} h_{-}(w(x+y)) d y=\frac{1}{\sigma_{-}} h_{-}(w(-\infty)) .
$$

(1.35) and (1.38) assure the existence of the limit $w^{\prime}(-\infty)=\lim _{x \rightarrow-\infty} w^{\prime}(x)$ and yield

$$
w^{\prime}(-\infty)=\frac{1}{\sigma_{-}} f(w(-\infty))
$$


This implies

$$
w(-\infty)=w^{\prime}(-\infty)=0
$$

(1.34) follows automatically from (1.40) (see [3] Chapter 13). This completes the proof of Lemma 1.2.

Suppose that $h_{-}(\xi)$ satisfies (1.32) and

$$
h_{-}\left(\xi_{0}\right)>0
$$

for some $\xi_{0} \in(0,1]$, then we have the more precise information than (1.34).

Lemma 1.3. If $\lambda>\lambda_{0}$ then there exist $a>0$ and $\delta>0$ such that

$$
\begin{gathered}
\left(\frac{d}{d x}\right)^{k} w(x)=\left(\sigma_{-}\right)^{k} a e^{\sigma_{-} x}\left\{1+O\left(e^{\delta x}\right)\right\} \quad \text { as } x \rightarrow-\infty \\
k=0,1,2, \\
\left\{\frac{w^{\prime \prime}(x)}{w^{\prime}(x)}\right\}^{\prime}=O\left(e^{\delta x}\right) \quad \text { as } x \rightarrow-\infty .
\end{gathered}
$$

\section{Lemma 1.4.}

If $\lambda=\lambda_{0}$, then there exist $a>0, b \in R^{1}$ and $\delta>0$ such that

$$
\begin{gathered}
\left(\frac{d}{d x}\right)^{k} w(x)=\left(\frac{d}{d x}\right)^{k}\left\{(b-a x) e^{\lambda_{0} x}\right\}\left\{1+O\left(e^{\delta x}\right)\right\} \quad \text { as } x \rightarrow-\infty \\
\left\{\frac{w^{\prime \prime}(x)}{w^{\prime}(x)}\right\}^{\prime}=O\left(\frac{1}{|x|^{2}}\right) \quad \text { as } x \rightarrow-\infty .
\end{gathered}
$$

Proof of Lemma 1.3. By (1.34) we have

$$
\int_{-\infty}^{0} e^{-\sigma_{-} y} h_{-}(w(y)) d y \quad<+\infty \text {. }
$$

Put

$$
\begin{aligned}
a & =-\frac{1}{\sigma_{+}-\sigma_{-}}\left\{u_{-}(0)-\int_{-\infty}^{0} e^{-\sigma_{-} y} h_{-}(w(y)) d y\right\}, \\
b & =\frac{1}{\sigma_{+}-\sigma_{-}} u_{+}(0) .
\end{aligned}
$$

It follows from (1.31) that

$$
\begin{aligned}
& u_{+}(x)=\left(\sigma_{+}-\sigma_{-}\right) b e^{\sigma_{+} x}-\int_{x}^{0} e^{\sigma_{+}(x-y)} h_{+}(w(y)) d y, \\
& u_{-}(x)=-\left(\sigma_{+}-\sigma_{-}\right) a e^{\sigma_{-} x}+\int_{-\infty}^{x} e^{\sigma_{-}(x-y)} h_{-}(w(y)) d y
\end{aligned}
$$

or equivalently 


$$
\begin{aligned}
& w(x)=a e^{\sigma_{-} x}+b e^{\sigma_{+} x}- \\
& -\frac{1}{\sigma_{+}-\sigma_{-}}\left\{\int_{x}^{0} e^{\sigma_{+}(x-y)} h_{+}(w(y)) d y+\int_{-\infty}^{x} e^{\sigma_{-}(x-y)} h_{-}(w(y)) d y\right\}, \\
& w^{\prime}(x)-g(w(x))=\sigma_{-} a e^{\sigma_{-} x}+\sigma_{+} b e^{\sigma_{+} x}- \\
& -\frac{1}{\sigma_{+}-\sigma_{-}}\left\{\int_{x}^{0} \sigma_{+} e^{\sigma_{+}(x-y)} h_{+}(w(y)) d y+\int_{-\infty}^{x} \sigma_{-} e^{\sigma_{-}(x-y)} h_{-}(w(y)) d y\right\} .
\end{aligned}
$$

(1.51) shows that $a \geqslant 0$. Suppose that $a=0$, then by (1.35) and (1.50) we have

$$
\int_{-\infty}^{\infty} e^{-\sigma_{-} y} h_{-}(w(y)) d y=0 \text {. }
$$

By (1.32) this implies

$$
h_{-}(w(x))=0 \quad x \in R^{1} .
$$

Since we have (1.41), this is a contradiction. This proves $a>0$. (1.42) follows at once from (1.51), (1.52) and (4). Note that

$$
\frac{u_{+}(x)}{w(x)}=O\left(e^{\delta x}\right) \quad \text { as } x \rightarrow-\infty
$$

for some $\delta>0$. Since we have

$$
\frac{w^{\prime \prime}}{w^{\prime}}=2 \lambda+g^{\prime}(w)-\frac{f(w)}{w^{\prime}},
$$

it follows

$$
\left(\frac{w^{\prime \prime}}{w^{\prime}}\right)^{\prime}=g^{\prime \prime}(w) w^{\prime}-f^{\prime}(w)+\frac{f(w) w^{\prime \prime}}{\left(w^{\prime}\right)^{2}}
$$

By (1.42) this implies

$$
\left(\frac{w^{\prime \prime}}{w^{\prime}}\right)^{\prime}=f^{\prime}(0)\left(\frac{w}{w^{\prime}}\right)^{2}\left(\frac{w^{\prime}}{w}\right)^{\prime}+O(w) \quad \text { as } x \rightarrow-\infty .
$$

Since

$$
\begin{aligned}
& w^{\prime}=\sigma_{-} w+g(w)+u_{+}, \\
& u_{+}^{\prime}=\sigma_{+} u_{+}+h_{+}(w),
\end{aligned}
$$

then it follows

$$
\begin{aligned}
& \left(\frac{w^{\prime}}{w}\right)^{\prime}=\left\{\sigma_{-}+\frac{g(w)}{w}+\frac{u_{+}}{w}\right\}^{\prime} \\
= & \left\{\frac{g^{\prime}(w)}{w}-\frac{g(w)}{w^{2}}\right\} w^{\prime}+\frac{\sigma_{+} u_{+}+h_{+}(w)}{w}- \\
- & \frac{u_{+}}{w^{2}}\left\{\sigma_{-} w+g(w)+u_{+}\right\} .
\end{aligned}
$$


Thus we have

$$
\left(\frac{w^{\prime}}{w}\right)^{\prime}=\left(\sigma_{+}-\sigma_{-}\right) \frac{u_{+}}{w}-\left(\frac{u_{+}}{w}\right)^{2}+O(w) \quad \text { as } x \rightarrow-\infty .
$$

By (1.42), (1.55), (1.58) and (1.61) we get (1.43). This proves Lemma 1.3.

Proof of Lemma 1.4. By (1.34) we have

$$
\int_{-\infty}^{0} e^{-\lambda_{0} y}|y|\left|h_{ \pm}(w(y))\right| d y \quad<+\infty .
$$

Put

$$
\begin{aligned}
& a=-\left\{u_{-}(0)-\int_{-\infty}^{0} e^{-\lambda_{0} y} h_{-}(w(y)) d y\right\} \\
& b=u_{+}(0)-\int_{-\infty}^{0} e^{-\lambda_{0} y}\left\{h_{+}(w(y))-y h_{-}(w(y))\right\} d y .
\end{aligned}
$$

It follows from (1.31) that

$$
\begin{aligned}
& u_{+}(x)=(b-a x) e^{\lambda_{0} x}+\int_{-\infty}^{x} e^{\lambda_{0}(x-y)}\left\{h_{+}(w(y))+(x-y) h_{-}(w(y))\right\} d y, \\
& u_{-}(x)=-a e^{\lambda_{0} x}+\int_{-\infty}^{x} e^{\lambda_{0}(x-y)} h_{-}(w(y)) d y
\end{aligned}
$$

or equivalently

$$
\begin{aligned}
& w(x)=(b-a x) e^{\lambda_{0} x}+\int_{-\infty}^{x} e^{\lambda_{0}(x-y)}\left\{h_{+}(w(y))+(x-y) h_{-}(w(y))\right\} d y, \\
& w^{\prime}(x)-g(w(x))=\left\{\lambda_{0}(b-a x)-a\right\} e^{\lambda_{0} x}+ \\
& +\int_{-\infty}^{x} e^{\lambda_{0}(x-y)}\left\{\lambda_{0} h_{+}(w(y))+\left(1+\lambda_{0}(x-y)\right) h_{-}(w(y))\right\} d y .
\end{aligned}
$$

(1.67) shows that $a \geqslant 0$. Suppose that $a=0$, then by (1.35) and (1.66) we have

$$
\int_{-\infty}^{\infty} e^{-\lambda_{0} y} h_{-}(w(y)) d y=0 \text {. }
$$

By (1.32) this implies

$$
h_{-}(w(x))=0 \quad x \in R^{1} .
$$

Since we have (1.41), this is a contradiction. This proves $a>0$. (1.44) follows at once from (1.67), (1.68) and (4). Now we proceed to prove (1.45). First we note that

$$
\frac{u_{-}(x)}{w(x)}=0\left(\frac{1}{|x|}\right) \quad \text { as } x \rightarrow-\infty .
$$

In this case we also have (1.58). Since 


$$
\begin{aligned}
& w^{\prime}=\lambda_{0} w+g(w)+u_{-}, \\
& u_{-}{ }^{\prime}=\lambda_{0} u_{-}+h_{-}(w) .
\end{aligned}
$$

Then it follows

$$
\left(\frac{w^{\prime}}{w}\right)^{\prime}=-\left(\frac{u_{-}}{w}\right)^{2}+O(w) \quad \text { as } x \rightarrow-\infty .
$$

By (1.44), (1.71), (1.58) and (1.74) we get (1.45). This completes the proof of Lemma 1.4. Thus we obtain

Theorem 1.2. We assume $\lambda \geqslant \lambda_{0}$. Suppose that $f$ satisfies the assumptions stated in Introduction, $g$ is a $C^{\infty}$ function satisfying (1.1), (1.32) and (1.41). Then there exists uniquely the solution $w(x)$ of (4) supplemented by the normalizing condition $w(0)=1 / 2$. $w(x)$ belongs to the class $M$ given by (7), and has the bounded continuous derivatives of any order. Moreover it satisfies

$$
\left\{\begin{array}{cl}
1-w(x)=a_{1} e^{\tau_{-} x}\left\{1+O\left(e^{-\delta x}\right\}\right) & \text { as } x \rightarrow+\infty \\
-w^{\prime}(x)=\tau_{-} a_{1} e^{\tau_{-} x}\left\{1+O\left(e^{-\delta x}\right)\right\} & \text { as } x \rightarrow+\infty
\end{array}\right.
$$

for some $a_{1}>0$ and $\delta>0$. Here $\tau_{-}=\tau_{-}(\mu)$ is given by (1.4) and (1.2). If $\lambda>\lambda_{0}$ then there exist $a>0$ and $\delta>0$ such that

$$
\begin{gathered}
\left(\frac{d}{d x}\right)^{k} w(x)=\left(\sigma_{-}\right)^{k} a e^{\sigma_{-} x}\left\{1+O\left(e^{\delta x}\right)\right\} \quad \begin{array}{r}
\text { as } x \rightarrow-\infty \\
k=0,1,2,
\end{array} \\
\left\{\frac{w^{\prime \prime}(x)}{w^{\prime}(x)}\right\}^{\prime}=O\left(e^{\delta x}\right) \quad \text { as } x \rightarrow-\infty .
\end{gathered}
$$

Here $\sigma_{-}=\sigma_{-}(\lambda)$ is given by (1.22). If $\lambda=\lambda_{0}$ then there exist $a>0, b \in R^{1}$ and $\delta>0$ such that

$$
\begin{gathered}
\left(\frac{d}{d x}\right)^{k} w(x)=\left(\frac{d}{d x}\right)^{k}\left\{(b-a x) e^{\lambda_{0} x}\right\}\left\{1+O\left(e^{\delta x}\right)\right\} \quad \begin{array}{l}
\text { as } x \rightarrow-\infty \\
k=0,1,2
\end{array} \\
\left\{\frac{w^{\prime \prime}(x)}{w^{\prime}(x)}\right\}^{\prime}=O\left(\frac{1}{|x|^{2}}\right) \quad \text { as } x \rightarrow-\infty .
\end{gathered}
$$

Since $g(\xi) \equiv 0$ satisfies (1.32) and (1.41) this theorem shows the existence and uniqueness of the solution $w_{\lambda}(x)$ of (2) supplemented by the normalizing condition $w(0)=1 / 2 . \quad w_{\lambda}(x)$ also satisfies $(1.75) \sim(1.79)$.

\section{KPP transform of the travelling wave}

In this section we investigate the properties of the KPP transform $\hat{w}(\xi)$ of the travelling wave $w(x)$. 
Theorem 2.1. If $\lambda \geqslant \lambda_{0}$, the KPP transform $\hat{w}(\xi)=w^{\prime}\left(w^{-1}(\xi)\right)$ of the travelling wave w(x) stated in Theorem 1.2 has the following properties:

$$
\begin{gathered}
\hat{w}(\xi) \in C^{1}[0,1] \cap C^{\infty}(0,1], \\
\hat{w}(\xi)>0 \quad \xi \in(0,1), \\
\hat{w}(0)=\hat{w}(1)=0, \quad \hat{w}^{\prime}(0)=\sigma_{-}(\lambda), \quad \hat{w}^{\prime}(1)=\tau_{-}(\mu),
\end{gathered}
$$

(Here $\sigma_{-}(\lambda)$ is given by (1.22), $\tau_{-}(\mu)$ is given by (1.4) and (1.2).)

$$
\begin{gathered}
\hat{w}^{\prime}(\xi)+\frac{f(\xi)}{\hat{w}(\xi)}=2 \lambda+g^{\prime}(\xi) \quad \xi \in(0,1), \\
\hat{w}^{2}(\xi)\left\{\hat{w}^{\prime \prime}(\xi)-g^{\prime \prime}(\xi)\right\}-f(\xi) \hat{w}^{\prime}(\xi)+f^{\prime}(\xi) \hat{w}(\xi)=0 \quad \xi \in(0,1), \\
\lim _{\xi \rightarrow+0} \xi \hat{w}^{\prime \prime}(\xi)=0
\end{gathered}
$$

Proof. It is easy to see (2.1) (2.3). (2.4) follows from (4). Differentiating (2.4) we get (2.5). Since

$$
\hat{w}(\xi) \hat{w}^{\prime \prime}(\xi)=\left.\left\{\frac{w^{\prime \prime}(x)}{w^{\prime}(x)}\right\}^{\prime}\right|_{x=w^{-1}(\xi)},
$$

by (1.43) or (1.45) we get (2.6). This completes the proof of theorem 2.1. It is easy to show

Lemma 2.1. Suppose that $\Omega(\xi)$ and $\omega(\xi)$ are both positive valued smooth functions on some interval $[\alpha, \beta] \subset[0,1]$. Then the differential inequality

$$
\left\{\begin{array}{l}
\omega^{\prime}(\xi)+\frac{f(\xi)}{\omega(\xi)}>\Omega^{\prime}(\xi)+\frac{f(\xi)}{\Omega(\xi)} \quad \xi \in(\alpha, \beta), \\
\omega(\beta)<\Omega(\beta)
\end{array}\right.
$$

yields

$$
\omega(\xi)<\Omega(\xi) \quad \xi \in[\alpha, \beta] .
$$

$\hat{w}_{\lambda}(\xi)$ satisfies $(2.1) \sim(2.6)$. Moreover it satisfies

\section{Theorem 2.2.}

$$
\begin{array}{ll}
\hat{w}_{\lambda_{2}}(\xi)<\hat{w}_{\lambda_{1}}(\xi) \quad \xi \in(0,1) & \lambda_{2}>\lambda_{1} \geqslant \lambda_{0}, \\
\lim _{\lambda \rightarrow \lambda_{1}} \sup _{0<\xi<1}\left|\hat{w}_{\lambda}(\xi)-\hat{w}_{\lambda_{1}}(\xi)\right|=0 & \lambda_{1} \geqslant \lambda_{0}, \\
\lim _{\lambda \rightarrow+\infty} \sup _{0<\xi<1} \hat{w}_{\lambda}(\xi)=0 . &
\end{array}
$$

Proof. Since $\hat{w}_{\lambda_{1}}(1)=\hat{w}_{\lambda_{2}}(1)=0, \hat{w}_{\lambda_{1}}{ }^{\prime}(1)=\tau_{-}\left(\lambda_{1}\right)<\tau_{-}\left(\lambda_{2}\right)=\hat{w}_{\lambda_{2}}{ }^{\prime}(1)$, then $(2.7)$ follows at once from (21) and Lemma 2.1. Multiplying (21) by $\hat{w}_{\lambda}(\xi)$, we get 


$$
\hat{w}_{\lambda}(\xi) \hat{w}_{\lambda}^{\prime}(\xi)+f(\xi)=2 \lambda \hat{w}_{\lambda}(\xi) \quad \xi \in(0,1) .
$$

Integrating over $(0, \xi)$ and imposing the boundary condition $\hat{w}_{\lambda}(0)=0$, we get

$$
\frac{1}{2} \hat{w}_{\lambda}^{2}(\xi)+\int_{0}^{\xi} f(\eta) d \eta=2 \lambda \int_{0}^{\xi} \hat{w}_{\lambda}(\eta) d \eta .
$$

Since $\hat{w}_{\lambda}(1)=0$, this implies

$$
\int_{0}^{1} \hat{w}_{\lambda}(\eta) d \eta=\frac{1}{2 \lambda} \int_{0}^{1} f(\eta) d \eta
$$

Suppose that $\lambda_{2}>\lambda_{1} \geqslant \lambda_{0}$, by (2.11) and (2.12) we get

$$
\begin{aligned}
& \frac{1}{2}\left\{\hat{w}_{\lambda_{1}}{ }^{2}(\xi)-\hat{w}_{\lambda_{2}}{ }^{2}(\xi)\right\} \leqslant 2 \lambda_{\lambda_{2}} \int_{0}^{\xi}\left\{\hat{w}_{\lambda_{1}}(\eta)-\hat{w}_{\lambda_{2}}(\eta)\right\} d \eta \leqslant \\
\leqslant & 2 \lambda_{2} \int_{0}^{1}\left\{\hat{w}_{\lambda_{1}}(\eta)-\hat{w}_{\lambda_{2}}(\eta)\right\} d \eta \\
= & \left(\frac{\lambda_{2}}{\lambda_{1}}-1\right) \int_{0}^{1} f(\eta) d \eta .
\end{aligned}
$$

This gives

$$
0 \leqslant \hat{w}_{\lambda_{1}}(\xi)-\hat{w}_{\lambda_{2}}(\xi) \leqslant\left(\frac{\lambda_{2}}{\lambda_{1}}-1\right) \int_{0}^{1} f(\eta) d \eta \frac{1}{\hat{w}_{\lambda_{2}}(\xi)} \quad \xi \in(0,1) .
$$

On the other hand we have

$$
0 \leqslant \hat{w}_{\lambda}(\xi) \leqslant \hat{w}_{\lambda_{0}}(\xi) \quad \xi \in[0,1] \quad \lambda \geqslant \lambda_{0} .
$$

(2.14) and (2.15) prove (2.8). Multiplying (2.10) by $\hat{w}_{\lambda}{ }^{\prime}(\xi)$, we get

$$
\hat{w}_{\lambda}(\xi)\left(\hat{w}_{\lambda}{ }^{\prime}(\xi)\right)^{2}=\left\{\lambda \hat{w}_{\lambda}{ }^{2}(\xi)-f(\xi) \hat{w}_{\lambda}(\xi)\right\}^{\prime}+f^{\prime}(\xi) \hat{w}_{\lambda}(\xi) .
$$

Integrating over $(0,1)$ and imposing the boundary condition $\hat{w}_{\lambda}(0)=\hat{w}_{\lambda}(1)=0$, we get

$$
\begin{aligned}
& \int_{0}^{1} \hat{w}_{\lambda}(\eta)\left(\hat{w}_{\lambda}^{\prime}(\eta)\right)^{2} d \eta=\int_{0}^{1} f^{\prime}(\eta) \hat{w}_{\lambda}(\eta) d \eta \leqslant \\
\leqslant & \sup _{0<\xi<1}\left|f^{\prime}(\xi)\right| \frac{1}{2 \lambda} \int_{0}^{1} f(\eta) d \eta .
\end{aligned}
$$

Integrating $(2.16)$ over $(0, \xi)$ and imposing $\hat{w}_{\lambda}(0)=0$, we get

$$
\begin{aligned}
& \lambda \hat{w}_{\lambda}{ }^{2}(\xi)-f(\xi) \hat{w}_{\lambda}(\xi)=\int_{0}^{\xi}\left\{\hat{w}_{\lambda}(\eta)\left(\hat{w}_{\lambda}{ }^{\prime}(\eta)\right)^{2}-f^{\prime}(\eta) \hat{w}_{\lambda}(\eta)\right\} d \eta \leqslant \\
\leqslant & \int_{0}^{1} \hat{w}_{\lambda}(\eta)\left(\hat{w}_{\lambda}{ }^{\prime}(\eta)\right)^{2} d \eta+\int_{0}^{1}\left|f^{\prime}(\eta)\right| \hat{w}_{\lambda}(\eta) d \eta .
\end{aligned}
$$

By (2.12) and (2.17) this gives 


$$
\lambda \hat{w}_{\lambda}^{2}(\xi)-f(\xi) \hat{w}_{\lambda}(\xi) \leqslant \frac{1}{\lambda} \sup _{0<\xi<1}\left|f^{\prime}(\xi)\right| \int_{0}^{1} f(\eta) d \eta .
$$

Since

$$
\lambda\left\{\hat{w}_{\lambda}(\xi)-\frac{1}{2 \lambda} f(\xi)\right\}^{2}=\lambda \hat{w}_{\lambda}{ }^{2}(\xi)-f(\xi) \hat{w}_{\lambda}(\xi)+\frac{1}{4 \lambda} f^{2}(\xi),
$$

It follows from (2.19) that

$$
\left\{\hat{w}_{\lambda}(\xi)-\frac{1}{2 \lambda} f(\xi)\right\}^{2} \leqslant \frac{1}{\lambda^{2}}\left\{\frac{1}{4} f^{2}(\xi)+\sup _{0<\xi<1}\left|f^{\prime}(\xi)\right| \int_{0}^{1} f(\eta) d \eta\right\} .
$$

This proves (2.9). This completes the proof of Theorem 2.2.

\section{Second existence theorem for the travelling wave}

Theorem 3.1. Suppose that $\lambda \geqslant \lambda_{0}$ and $g$ satisfies (1.1). We assume that there exists smooth function $\hat{W}(\xi)$ defined on $[0,1]$ which satisfies

$$
\begin{aligned}
& \hat{W}^{\prime}(\xi)+\frac{f(\xi)}{W(\xi)}<2 \lambda+g^{\prime}(\xi) \quad \xi \in(0,1), \\
& \hat{W}(\xi)>0 \quad \xi \in(0,1), \quad \hat{W}(0)=0, \\
& \hat{W}(1)=0, \quad \hat{W}^{\prime}(1)<\tau_{-}(\mu) \quad(\text { or } \hat{W}(1)>0) .
\end{aligned}
$$

Here $\tau_{-}(\mu)$ is given by (1.4) and (1.2). Then there exists uniquely the solution $w(x)$ of (4) supplemented by the normalizing condition $w(0)=1 / 2$. $\hat{w}(\xi)$ satisfies $(2.1)$, (2.2), (2.4), (2.5) and

$$
\begin{array}{ll}
\hat{w}(0)=\hat{w}(1)=0, & \hat{w}^{\prime}(1)=\tau_{-}(\mu) . \\
\hat{w}^{\prime}(0)=\sigma_{-}(\lambda) \quad \text { or } \quad \sigma_{+}(\lambda) .
\end{array}
$$$$
\xi \hat{w}^{\prime \prime}(\xi) \text { is bounded on }[0,1] \text {. }
$$

Here $\tau_{-}(\mu)$ is given by (1.4) and (1.2), and $\sigma_{ \pm}(\lambda)$ is given by (1.22).

Proof. Let us fix $\lambda_{1}>\lambda_{0}$ such that

$$
2 \lambda+g^{\prime}(\xi)<2 \lambda_{1} \quad \xi \in[0,1] .
$$

By Lemma 1.1 we have the solution $\hat{w}(\xi)$ of

$$
\hat{w}^{\prime}(\xi)+\frac{f(\xi)}{\hat{w}(\xi)}=2 \lambda+g^{\prime}(\xi)
$$

defined in some subinterval $\left[\xi_{1}, 1\right]$ of $[0,1]$ which satisfies

$$
\hat{w}(1)=0, \quad \hat{w}^{\prime}(1)=\tau_{1}(\mu), \quad \hat{w}(\xi)>0 \quad \xi \in\left[\xi_{1}, 1\right) .
$$


Lemma 2.1 assures the inequality

$$
\hat{w}_{\lambda_{1}}(\xi)<\hat{w}(\xi)<\hat{W}(\xi)
$$

as long as $\hat{w}(\xi)$ satisfies (3.8). Thus we can continue $\hat{w}(\xi)$ on $[0,1]$ as a solution of (3.8). The relation

$$
x=\int_{1 / 2}^{w(x)} \frac{d \eta}{\hat{w}(\eta)} \quad x \in R^{1}
$$

gives the solution $w(x)$ of $(4)$ with $w(0)=1 / 2$. We omit the proof of the remaining parts. It can be easily obtained by the standard theory of 2-dimensional autonomous system.

\section{Part II. Asymptotic Behavior of the Time Dependent Solution}

\section{Stability and instability criteria for $w_{\lambda}(x)$}

Throughout this section we assume $\lambda \geqslant \lambda_{0}$. We study the relation between the solutions of the time dependent problem

$$
\left\{\begin{array}{l}
{\left[L+2 \lambda \frac{\partial}{\partial x}\right] u=f(u), \quad 0 \leqslant u \leqslant 1 \quad(x, t) \in R^{1} \times(0, \infty),} \\
u(x, 0)=u_{0}(x) \quad x \in R^{1}
\end{array}\right.
$$

and the travelling wave $w_{\lambda}(x)$. As is proved in Part $\mathrm{I} w_{\lambda}(x)$ is the unique solution of the problem

$$
w^{\prime \prime}-2 \lambda w^{\prime}+f(w)=0, \quad 0 \leqslant w \leqslant 1 \quad x \in R^{1}
$$

supplemented by the normalizing condition $w(0)=1 / 2$. Throughout this section $\nu>0$ is taken such that the function $F(\xi ; \nu)$ given by

$$
F(\xi ; \nu)=f(\xi)+\nu \xi \quad \xi \in[0,1]
$$

is monotone increasing with respect to $\xi$ in $[0,1]$. Let us introduce following notations:

$$
\begin{gathered}
H(x, t)=\frac{1}{\sqrt{4 \pi t}} e^{-x^{2} / 4 t}, \\
K(x ; \mu)=\int_{0}^{\infty} e^{-\mu t} H(x, t) d t=\frac{1}{2 \sqrt{\mu}} e^{-\sqrt{\mu}|x|} \quad(\mu>0), \\
U(x, y, t ; \lambda, \nu)=e^{\lambda(x-y)-\left(\lambda^{2}+\nu\right) t} H(x-y, t) \\
=e^{-\nu t} H(x-2 \lambda t-y, t), \\
U_{-}(x, y, t ; \lambda, \nu)=e^{\lambda(x-y)-\left(\lambda^{2}+\nu\right) t}\{H(x-y, t)-H(x+y, t)\},
\end{gathered}
$$




$$
\begin{aligned}
G(x, y ; \lambda, \nu) & =e^{\lambda(x-y)} K\left(x-y ; \lambda^{2}+\nu\right), \\
G_{-}(x, y ; \lambda, \nu) & =e^{\lambda(x-y)}\left\{K\left(x-y ; \lambda^{2}+\nu\right)-K\left(x+y ; \lambda^{2}+\nu\right)\right\} .
\end{aligned}
$$

It is easy to see the following relations:

$$
\begin{aligned}
& \int_{-\infty}^{\infty} U(x, y, t ; \lambda, \nu) d y=e^{-\nu t}, \\
& \int_{t}^{\infty} \int_{-\infty}^{\infty} U(x, y, s ; \lambda, \nu) d y d s=\frac{1}{\nu} e^{-\nu t},
\end{aligned}
$$$$
\int_{-\infty}^{\infty} U(x, y, t ; \lambda, \nu) d y+\nu \int_{0}^{t} \int_{-\infty}^{\infty} U(x, y, s ; \lambda, \nu) d y d s=1 \text {, }
$$$$
\int_{-\infty}^{\infty} G(x, y ; \lambda, \nu) d y=\frac{1}{\nu} \text {, }
$$$$
\int_{0}^{\infty} U(x, y, t ; \lambda, \nu) d t=G(x, y ; \lambda, \nu) \text {, }
$$$$
U_{-}(x, y, t ; \lambda, \nu)>0 \quad(x, y, t) \in(-\infty, 0) \times(-\infty, 0) \times(0, \infty),
$$$$
\left[-\frac{\partial}{\partial y} U_{-}(x, y, t ; \lambda, \nu)\right]_{y=0}>0 \quad(x, t) \in(-\infty, 0) \times(0, \infty) \text {, }
$$

$$
G_{-}(x, y ; \lambda, \nu)>0 \quad(x, y) \in(-\infty, 0) \times(-\infty, 0) .
$$

Suppose that

$$
\left\{\begin{array}{l}
\omega(x) \in \mathscr{B}^{2}(-\infty, 0], \quad \omega(0)=1, \\
0 \leqslant \omega(x) \leqslant 1 \quad x \leqslant 0 .
\end{array}\right.
$$

Define $\tilde{\omega}(x)$ by the relation

$$
\tilde{\omega}(x)=-\omega^{\prime \prime}(x)+2 \lambda \omega^{\prime}(x)-\lambda_{0}^{2} \omega(x) \quad x \leqslant 0 .
$$

Then we have

$$
\left[-\left(\frac{d}{d x}\right)^{2}+2 \lambda \frac{d}{d x}+\nu\right] \omega=\left(\lambda_{0}^{2}+\nu\right) \omega+\tilde{\omega} \quad x<0 .
$$

By (4.16) and (4.18), we have

$$
\begin{aligned}
& \omega(x)=e^{\rho_{+} x}+ \\
& +\int_{-\infty}^{0} G_{-}(x, y ; \lambda, \nu)\left\{\left(\lambda_{0}^{2}+\nu\right) \omega(y)+\tilde{\omega}(y)\right\} d y \quad x \leqslant 0 .
\end{aligned}
$$

Here we use the abbreviation

$$
\rho_{+}=\lambda+\sqrt{\lambda^{2}+\nu} .
$$

It can be also represented as 


$$
\begin{aligned}
& \omega(x)=\int_{-\infty}^{0} U_{-}(x, y, t ; \lambda, \nu) \omega(y) d y+ \\
& +\int_{0}^{t} \int_{-\infty}^{0} U_{-}(x, y, t-s ; \lambda, \nu)\left\{\left(\lambda_{0}^{2}+\nu\right) \omega(y)+\tilde{\omega}(y)\right\} d y d s+ \\
& +\int_{0}^{t}\left[-\frac{\partial}{\partial y} U_{-}(x, y, t-s ; \lambda, \nu)\right]_{y=0} d s .
\end{aligned}
$$

We define $\left\{\bar{w}_{j}(x)\right\}_{j=0}^{\infty}$ by

$$
\begin{gathered}
\bar{w}_{0}(x)=\left\{\begin{array}{cc}
1 & x \geqslant 0, \\
\omega(x) & x \leqslant 0
\end{array}\right. \\
\bar{w}_{j}(x)=\int_{-\infty}^{\infty} G(x, y ; \lambda, \nu) F\left(\bar{w}_{j-1}(y) ; \nu\right) d y \quad j=1,2,3, \cdots .
\end{gathered}
$$

We define $\left\{\boldsymbol{u}_{j}(x, t)\right\}_{j=0}^{\infty}$ by

$$
\begin{aligned}
& u_{0}(x, t)=\bar{w}_{0}(x) \\
& u_{j}(x, t)=\int_{-\infty}^{\infty} U(x, y, t ; \lambda, \nu) \bar{w}_{0}(y) d y+ \\
& +\int_{0}^{t} \int_{-\infty}^{\infty} U(x, y, t-s ; \lambda, \nu) F\left(\bar{u}_{j-1}(y, s) ; \nu\right) d y d s \quad j=1,2,3, \cdots .
\end{aligned}
$$

Hereafter we assume

$$
\tilde{\omega}(x) \geqslant 0 \quad x \leqslant 0
$$

\section{Lemma 4.1.}

$$
\begin{aligned}
& 0<\bar{w}_{1}(x)<\bar{w}_{0}(x) \quad x \in R^{1}, \\
& 0<\bar{u}_{1}(x, t)<\bar{u}_{0}(x, t)=\bar{w}_{0}(x) \quad(x, t) \in R^{1} \times(0, \infty), \\
& \bar{u}_{1}(x, t+h)<\bar{u}_{1}(x, t) \quad(x, t) \in R^{1} \times(0, \infty), \quad h>0 .
\end{aligned}
$$

Proof. (4.26) shows that $\bar{w}_{0}(x) \neq 1$ it follows from (4.11) and (4.23) that

$$
0<\bar{w}_{1}(x)<1 \quad x \in R^{1} .
$$

To prove (4.27) it suffices to show

$$
0<\bar{w}_{1}(x)<\omega(x) \quad x \leqslant 0 .
$$

By (4.23) we have

$$
\left[-\left(\frac{d}{d x}\right)^{2}+2 \lambda \frac{d}{d x}+\nu\right] \bar{w}_{1}=F(\omega(x) ; \nu) \quad x<0 .
$$

Therefore $\bar{w}_{1}(x)$ can be represented as 


$$
\bar{w}_{1}(x)=\bar{w}_{1}(0) e^{\rho_{+}} x+\int_{-\infty}^{0} G_{-}(x, y ; \lambda, \nu) F(\omega(y) ; \nu) d y .
$$

Here $\rho_{+}$is given by (4.20). Since $\bar{w}_{1}(0)<1$ and $F(\omega(y) ; \nu) \leqslant\left(\lambda_{0}^{2}+\nu\right) \omega(y)$ for $y<0$, it follows from the positivity of the kernel $G_{-}$that

$$
\bar{w}_{1}(x)<e^{\rho_{+}}{ }^{x}+\int_{-\infty}^{0} G_{-}(x, y ; \lambda, \nu)\left(\lambda_{0}^{2}+\nu\right) \omega(y) d y .
$$

(4.26), (4.34) and (4.19) give (4.31). This proves (4.27). It is easy to see

$$
0<n_{1}(x, t)<1 \quad(x, t) \in R^{1} \times(0, \infty) .
$$

To prove (4.28), it suffices to show

$$
0<u_{1}(x, t)<\omega(x) \quad(x, t) \in(-\infty, 0] \times(0, \infty) .
$$

By (4.25) we have

$$
\left\{\begin{array}{l}
{\left[L+2 \lambda \frac{\partial}{\partial x}+\nu\right] \vec{u}_{1}=F(\omega(x) ; \nu) \quad(x, t) \in(-\infty, 0) \times(0, \infty),} \\
a_{1}(x, 0)=\omega(x) \quad x \in(-\infty, 0) .
\end{array}\right.
$$

So we have

$$
\begin{aligned}
& a_{1}(x, t)=\int_{-\infty}^{0} U_{-}(x, y, t ; \lambda, \nu) \omega(y) d y+ \\
& +\int_{0}^{t} \int_{-\infty}^{0} U_{-}(x, y, t-s ; \lambda, \nu) F(\omega(y) ; \nu) d y d s+ \\
& +\int_{0}^{t}\left[-\frac{\partial}{\partial y} U_{-}(x, y, t-s ; \lambda, \nu)\right]_{y=0} a_{1}(0, s) d s .
\end{aligned}
$$

This gives

$$
\begin{aligned}
& a_{1}(x, t)<\int_{-\infty}^{0} U_{-}(x, y, t ; \lambda, \nu) \omega(y) d y+ \\
& +\int_{0}^{t} \int_{-\infty}^{0} U_{-}(x, y, t-s ; \lambda, \nu)\left(\lambda_{0}^{2}+\nu\right) \omega(y) d y d s+ \\
& +\int_{0}^{t}\left[-\frac{\partial}{\partial y} U_{-}(x, y, t-s ; \lambda, \nu)\right]_{y=0} d s .
\end{aligned}
$$

(4.26), (4.39) and (4.21) show (4.36). This proves (4.28). For any $h>0$ we have

$$
\begin{aligned}
& u_{1}(x, t+h)=\int_{-\infty}^{\infty} U(x, y, t ; \lambda, \nu) u_{1}(y, h) d y+ \\
& +\int_{0}^{t} \int_{-\infty}^{\infty} U(x, y, t-s ; \lambda, \nu) F\left(\bar{w}_{0}(y) ; \nu\right) d y d s .
\end{aligned}
$$

By (4.28) we have 


$$
\begin{aligned}
& u_{1}(x, t+h)<\int_{-\infty}^{\infty} U(x, y, t ; \lambda, \nu) \bar{w}_{0}(y) d y+ \\
& +\int_{0}^{t} \int_{-\infty}^{\infty} U(x, y, t-s ; \lambda, \nu) F\left(\bar{w}_{0}(y) ; \nu\right) d y d s .
\end{aligned}
$$

By (4.25) this gives (4.29). This completes the proof of Lemma 4.1.

\section{Lemma 4.2.}

$$
\begin{aligned}
& \bar{w}_{j-1}(x) \geqslant \bar{w}_{j}(x)>0 \quad x \in R^{1} \quad j=1,2,3, \cdots, \\
& \boldsymbol{u}_{j-1}(x, t) \geqslant \pi_{j}(x, t)>0 \quad(x, t) \in R^{1} \times(0, \infty) \quad j=1,2,3, \cdots, \\
& \boldsymbol{u}_{j}(x, t) \geqslant \pi_{j}(x, t+h) \quad(x, t) \in R^{1} \times(0, \infty), \quad h>0, \quad j=1,2,3, \cdots .
\end{aligned}
$$

Proof. (4.42) and (4.43) follow from (4.27) and (4.28) by using the monotonicity of the nonlinearity and the positity of the kernels. For any $h>0$ we have

$$
\begin{aligned}
& \boldsymbol{u}_{j}(x, t+h)=\int_{-\infty}^{\infty} U(x, y, t ; \lambda, \nu) \boldsymbol{u}_{j}(y, h) d y+ \\
& +\int_{0}^{t} \int_{-\infty}^{\infty} U(x, y, t-s ; \lambda, \nu) F\left(\boldsymbol{u}_{j-1}(y, s+h) ; \nu\right) d y d s \quad j=1,2,3, \cdots .
\end{aligned}
$$

(4.28) and (4.43) give

$$
\boldsymbol{u}_{j}(y, h)<\bar{w}_{0}(y) \quad j=1,2,3, \cdots .
$$

(4.46) and the induction hypothesis

$$
\boldsymbol{u}_{j-1}(y, s+h) \leqslant \boldsymbol{u}_{j-1}(y, s) \quad(y, s) \in R^{1} \times(0, \infty)
$$

give

$$
\begin{aligned}
& u_{j}(x, t+h) \leqslant \int_{-\infty}^{\infty} U(x, y, t ; \lambda, \nu) \bar{w}_{0}(y) d y+ \\
& +\int_{0}^{t} \int_{-\infty}^{\infty} U(x, y, t-s ; \lambda, \nu) F\left(u_{j-1}(y, s) ; \nu\right) d y d s= \\
& =u_{j}(x, t) .
\end{aligned}
$$

This proves (4.44). This completes the proof of Lemma 4.2.

Lemma 4.3. For any $j \geqslant 0$ we have

$$
\lim _{t \rightarrow \infty} \pi_{j}(x, t)=\bar{w}_{j}(x)
$$

uniformly with respect to $x \in R^{1}$, monotone decreasingly with respect to $t$.

Proof. By (4.12) and (4.23) we have

$$
\bar{w}_{i}(x)=\int_{0}^{t} \int_{-\infty}^{\infty} U(x, y, t-s ; \lambda, \nu) F\left(\bar{w}_{j-1}(y) ; \nu\right) d y d s+
$$




$$
+\int_{t}^{\infty} \int_{-\infty}^{\infty} U(x, y, s ; \lambda, \nu) F\left(\bar{w}_{j-1}(y) ; \nu\right) d y d s .
$$

Subtracting (4.50) from (4.25) we have

$$
\vec{u}_{j}(x, t)-\bar{w}_{j}(x)=I_{1}+I_{2}+I_{3}+I_{4} .
$$

Here

$$
\begin{aligned}
I_{1}= & \int_{-\infty}^{\infty} U(x, y, t ; \lambda, \nu) \bar{w}_{0}(y) d y, \\
I_{2}= & -\int_{t}^{\infty} \int_{-\infty}^{\infty} U(x, y, s ; \lambda, \nu) F\left(\bar{w}_{j-1}(y) ; \nu\right) d y d s, \\
I_{3}= & \int_{t-T}^{t} \int_{-\infty}^{\infty} U(x, y, s ; \lambda, \nu)\left\{F\left(u_{j-1}(y, t-s) ; \nu\right)-\right. \\
& \left.-F\left(\bar{w}_{j-1}(y) ; \nu\right)\right\} d y d s, \\
I_{4}= & \int_{T}^{t} \int_{-\infty}^{\infty} U(x, y, t-s ; \lambda, \nu)\left\{F\left(u_{j-1}(y, s) ; \nu\right)-\right. \\
& \left.-F\left(\bar{w}_{j-1}(y) ; \nu\right)\right\} d y d s \quad(0<T<t) .
\end{aligned}
$$

By (4.8), (4.9), (4.11) and (4.12) we have

$$
\begin{aligned}
& \left|I_{1}\right| \leqslant e^{-\nu t}, \\
& \left|I_{2}\right| \leqslant e^{-\nu t}, \\
& \left|I_{3}\right| \leqslant e^{-\nu(t-T)}, \\
& \left|I_{4}\right| \leqslant \frac{1}{\nu} \sup _{0<\xi<1}\left|F^{\prime}(\xi, \nu)\right| \sup _{\substack{y \in R^{1} \\
s>r}}\left|u_{j-1}(y, s)-\bar{w}_{j-1}(y)\right| .
\end{aligned}
$$

This proves (4.49) inductively. This completes the proof of Lemma 4.3. Lemma 4.2 shows the existence of the limits

$$
\begin{aligned}
& \bar{w}(x)=\lim _{j \rightarrow \infty} \bar{w}_{j}(x), \\
& \vec{u}(x, t)=\lim _{j \rightarrow \infty} \bar{n}_{i}(x, t) .
\end{aligned}
$$

Letting $j$ tends to infinity in (4.23) and (4.25) we have

$$
\begin{aligned}
& \bar{w}(x)=\int_{-\infty}^{\infty} G(x, y ; \lambda, \nu) F(\bar{w}(y) ; \nu) d y, \\
& u(x, t)=\int_{-\infty}^{\infty} U(x, y, t ; \lambda, \nu) \bar{w}_{0}(y) d y+ \\
& +\int_{0}^{t} \int_{-\infty}^{\infty} U(x, y, t-s ; \lambda, \nu) F(u(y, s) ; \nu) d y d s .
\end{aligned}
$$

So we have 


$$
\begin{aligned}
& {\left[-\left(\frac{d}{d x}\right)^{2}+2 \lambda \frac{d}{d x}+\nu\right] \bar{w}(x)=F(\bar{w}(x) ; \nu),} \\
& \left\{\begin{array}{l}
{\left[L+2 \lambda \frac{\partial}{\partial x}+\nu\right] \bar{u}(x, t)=F(u(x, t) ; \nu),} \\
u(x, 0)=\bar{w}_{0}(x) .
\end{array}\right.
\end{aligned}
$$

Finally we have

\section{Lemma 4.4.}

$$
\begin{aligned}
& \bar{w}^{\prime \prime}-2 \lambda \bar{w}^{\prime}+f(\bar{w})=0, \quad 0 \leqslant \bar{w}<1 \quad x \in R^{1}, \\
& \begin{cases}{\left[L+2 \lambda \frac{\partial}{\partial x}\right] \bar{u}=f(u), \quad 0 \leqslant u<1} & (x, t) \in R^{1} \times(0, \infty), \\
u(x, 0)=\bar{w}_{0}(x) \quad x \in R^{1} . & \end{cases}
\end{aligned}
$$

Since $\bar{w}(x)$ is continuous it follows

\section{Lemma 4.5.}

$$
\lim _{j \rightarrow \infty} \bar{w}_{j}(x)=\bar{w}(x)
$$

uniformly with respect to $x$ in every finite subinterval of $R^{1}$ and monotone decreasingly with respect to $j$.

Since $u(x, t)$ is continuous, it follows

\section{Lemma 4.6.}

$$
\lim _{j \rightarrow \infty} u_{j}(x, t)=u(x, t)
$$

uniformly with respect to $(x, t)$ in every compact subset of $R^{1} \times[0, \infty)$ and monotone decreasingly with respect to $j$.

(4.44) gives

\section{Lemma 4.7.}

$$
u(x, t) \geqslant u(x, t+h) \quad(x, t) \in R^{1} \times[0, \infty), \quad h>0 .
$$

By (4.49), (4.68), (4.69) and (4.70) we have

\section{Lemma 4.8.}

$$
\lim _{t \rightarrow \infty} \bar{u}(x, t)=\bar{w}(x)
$$

uniformly with respect to $x$ in every finite subinterval of $R^{1}$ and monotone decreasingly with respect to $t$.

Theorem 4.1. We assume $\lambda \geqslant \lambda_{0}$. Suppose that the solution $u(x, t)$ of $(5)$ 
has the initial function

$$
\bar{w}_{0}(x)=\left\{\begin{array}{cc}
1 & x \geqslant 0, \\
\omega(x) & x \leqslant 0 .
\end{array}\right.
$$

Here $\omega(x)$ satisfies

$$
\begin{gathered}
\left\{\begin{array}{l}
\omega(x) \in \mathscr{B}^{2}(-\infty, 0], \quad \omega(0)=1, \\
0 \leqslant \omega(x) \leqslant 1 \quad x \leqslant 0 .
\end{array}\right. \\
\tilde{\omega}(x)=-\omega^{\prime \prime}(x)+2 \lambda \omega^{\prime}(x)-\lambda_{0}^{2} \omega(x) \geqslant 0 \quad x \leqslant 0
\end{gathered}
$$

and

$$
\lim _{x \rightarrow-\infty} \inf \frac{\omega(x)}{w_{\lambda}(x)}=0
$$

Then we have

$$
\lim _{t \rightarrow \infty} \pi(x, t)=0
$$

uniformly with respect to $x$ in every finite subinterval of $R^{1}$ and monotone decreasingly with respect to $t$. If we and the assumption $\omega(-\infty)=0$, then the convergence in (4.73) is uniform with respect to $x \in(-\infty, A]$ for any $A$.

Proof. By (4.71) it suffices to prove $\bar{w}(x)=0$. Suppose that this is not true, then by (4.66) there exists $c$ such that $\bar{w}(x)=w_{\lambda}(x+c)$. Since $\bar{w}(x) \leqslant \omega(x) x \leqslant 0$, we have

$$
0=\lim _{x \rightarrow-\infty} \inf \frac{\omega(x)}{w_{\lambda}(x)} \geqslant \lim _{x \rightarrow-\infty} \inf \frac{w_{\lambda}(x+c)}{w_{\lambda}(x)} .
$$

On the other hand, by (1.76) or (1.78) we have

$$
\lim _{x \rightarrow-\infty} \frac{w_{\lambda}(x+c)}{w_{\lambda}(x)}>0
$$

This is a contradiction. Therefore we have $\bar{w}(x)=0$. This completes the proof of Theorem 4.1. By comparison theorem we have

Corollary to Theorem 4.1. Suppose that the solution $u(x, t)$ of $(5)$ has the initial function $u_{0}(x)$. If

$$
0 \leqslant u_{0}(x) \leqslant \bar{w}_{0}(x) \quad x \in R^{1}
$$

with $\bar{w}_{0}(x)$ given in Theorem 4.1, then we have

$$
\lim _{t \rightarrow \infty} u(x, t)=0
$$

uniformly with respect to $x$ in every finite subinterval of $R^{1}$. In case of $\omega(-\infty)=0$, the above convergence is uniform with respect to $x \in(-\infty, A]$ for any $A$. 
Now we give some examples of $\omega(x)$ which satisfy all the conditions stated in Theorem 4.1.

EXAMPLE 4.1. $\left(\lambda>\lambda_{0}\right)$

$$
\omega(x)=e^{\sigma^{x}} \quad \sigma_{-}(\lambda)<\sigma \leqslant \sigma_{+}(\lambda) .
$$

EXAMPLE 4.2. $\left(\lambda>\lambda_{0}\right)$

$$
\omega(x)=e^{\sigma_{-}(\lambda) x}(1-\alpha x)^{-\beta} .
$$

Here $\alpha>0, \beta>0$ and $\alpha(1+\beta) \leqslant \sigma_{+}-\sigma_{-}$.

EXAMPLE 4.3. $\left(\lambda>\lambda_{0}\right)$

$$
\omega(x)=e^{\sigma_{-}(\lambda) x} \frac{1}{1+l_{n+1}(-\alpha x)} .
$$

Here $\alpha>0, \beta>0, \alpha\left(1+\beta+\cdots+\beta^{n}+2 \beta^{n+1}\right) \leqslant \sigma_{+}-\sigma_{-}$and

$$
\left\{\begin{array}{l}
l_{1}(x)=\beta \log (1+x), \\
l_{j+1}(x)=l_{1}\left(l_{j}(x)\right) \quad j=1,2, \cdots, n .
\end{array}\right.
$$

EXAMPLE 4.4. $\left(\lambda=\lambda_{0}\right)$

$$
\omega(x)=e^{\lambda_{0} x}\left(1-\tilde{\lambda}_{x} x\right)\left(1-\alpha \tilde{\lambda}_{2} x\right)^{-\beta} .
$$

Here $\lambda_{0} \geqslant \tilde{\lambda}>0,1 \geqslant \alpha>0$ and $1 \geqslant \beta>0$.

EXAMPLE 4.5. $\left(\lambda=\lambda_{0}\right)$

$$
\omega(x)=e^{\lambda_{0} x} \frac{1-\tilde{\lambda} x}{1+l_{n+1}(-\alpha \tilde{\lambda} x)} .
$$

Here $\lambda_{0} \geqslant \tilde{\lambda}>0,1 \geqslant \alpha>0, \beta>0, \beta+\cdots+\beta^{n}+2 \beta^{n+1} \leqslant 1$ and $l_{n+1}(x)$ is given by (4.77).

Suppose that $k(x)$ satisfies

$$
k(0)=1, k(+\infty)=0, k^{\prime}(x)<0 \quad x \geqslant 0 .
$$

Put

$$
\omega(x)= \begin{cases}e^{\sigma_{-}(\lambda) x} k(-\alpha x) & \left(\lambda>\lambda_{0}\right), \\ e^{\lambda_{0} x}(1-\tilde{\lambda} x) k(-\alpha \tilde{\lambda} x) & \left(\lambda=\lambda_{0}\right) .\end{cases}
$$

Here $\alpha>0$ and $\lambda_{0} \geqslant \tilde{\lambda}>0$. Since

$$
\text { (4.82) } \tilde{\omega}(x)=\left\{\begin{array}{l}
-e^{\sigma_{-} x} \alpha^{2} k^{\prime}(-\alpha x)\left\{\frac{\sigma_{+}-\sigma_{-}}{\alpha}+\frac{k^{\prime \prime}(-\alpha x)}{k^{\prime}(-\alpha x)}\right\} \quad\left(\lambda>\lambda_{0}\right), \\
-e^{\lambda_{0} x}(1-\tilde{\lambda} x)(\alpha \tilde{\lambda})^{2} k^{\prime}(-\alpha \tilde{\lambda} x)\left\{\frac{2}{\alpha-\alpha \tilde{\lambda} x}+\frac{k^{\prime \prime}(-\alpha \tilde{\lambda} x)}{k^{\prime}(-\alpha \tilde{\lambda} x)}\right\} \quad\left(\lambda=\lambda_{0}\right) .
\end{array}\right.
$$


Then we have

$$
\tilde{\omega}(x) \geqslant 0 \quad x \leqslant 0
$$

if and only if

$$
-\frac{k^{\prime \prime}(x)}{k^{\prime}(x)} \leqslant \begin{cases}\frac{\sigma_{+}-\sigma_{-}}{\alpha} & \left(\lambda>\lambda_{0}\right), \\ \frac{2}{\alpha+x} & \left(\lambda=\lambda_{0}\right) \quad x \geqslant 0 .\end{cases}
$$

It is easy to see that

$$
k(x)=\frac{1}{1+l_{n+1}(x)}
$$

satisfies (4.80) and

$$
-\frac{k^{\prime \prime}(x)}{k^{\prime}(x)}=\frac{2 l_{n+1}^{\prime}(x)}{1+l_{n+1}(x)}-\frac{l_{n+1}^{\prime \prime}(x)}{l_{n+1}^{\prime}(x)}
$$

Since

$$
0<\frac{2 l_{n+1}^{\prime}(x)}{1+l_{n+1}(x)}-\frac{l_{n+1}{ }^{\prime \prime}(x)}{l_{n+1}{ }^{\prime}(x)} \leqslant \frac{1+\beta+\cdots+\beta^{n}+2 \beta^{n+1}}{1+x} .
$$

Then under conditions stated in Examples 4.3 or $4.5, k(x)$ given by (4.85) satisfies (4.84). This shows that $\omega(x)$ in Examples 4.3 or 4.5 satisfies (4.83). All the other requirements in Theorem 4.1 are also satisfied. Especially we have $\omega(-\infty)=0$. The above examples give fairly sharp instability criteria for the travelling wave $w_{\lambda}(x)$. Now we proceed to show a stability criteron for the travelling wave $w_{\lambda}(x)$.

Theorem 4.2. Suppose that the solution $\vec{u}(x, t)$ of (5) has the initial function

$$
\bar{w}_{0}(x)=\left\{\begin{array}{cc}
1 & x \geqslant 0, \\
\omega(x) & x \leqslant 0
\end{array}\right.
$$

with

$$
\omega(x)= \begin{cases}e^{\sigma_{-} x} & \left(\lambda>\lambda_{0}\right), \\ e^{\lambda_{0} x}(1-\tilde{\lambda} x) & \left(\lambda=\lambda_{0}\right) .\end{cases}
$$

Here $\lambda_{0} \geqslant \tilde{\lambda}>0$. Then there exists $c$ such that

$$
\lim _{t \rightarrow \infty} u(x, t)=w_{\lambda}(x+c)
$$

uniformly with respect to $x \in R^{1}$ and monotone decreasingly with respect to $t$.

Proof. Note that $\tilde{\omega}(x)=0 x \leqslant 0$. By (4.71) and (4.66) it suffices to show that $\bar{w}(x) \neq 0$. By (1.76) or (1.78) there exists $c_{1}$ such that 


$$
\bar{w}_{0}(x) \geqslant w_{\lambda}\left(x+c_{1}\right) \quad x \in R^{1} .
$$

Since we have

$$
w_{\lambda}\left(x+c_{1}\right)=\int_{-\infty}^{\infty} G(x, y ; \lambda, \nu) F\left(w_{\lambda}\left(y+c_{1}\right) ; \nu\right) d y,
$$

it follows

$$
\bar{w}_{j}(x) \geqslant w_{\lambda}\left(x+c_{1}\right) \quad x \in R^{1} \quad j=0,1,2, \cdots .
$$

This gives

$$
1>\bar{w}(x) \geqslant w_{\lambda}\left(x+c_{1}\right) \quad x \in R^{1} .
$$

This proves Theorem 4.2. Theorem 4.2 and the comparison theorem give

Corollary to Theorem 4.2. Suppose that the solution $u(x, t)$ of $(5)$ has the initial function $u_{0}(x)$. If

$$
w_{\lambda}\left(x+c_{1}\right) \leqslant u_{0}(x) \leqslant \bar{w}_{0}\left(x+c_{2}\right) \quad x \in R^{1}
$$

for some $c_{1}$ and $c_{2}$, where $\bar{w}_{0}(x)$ is the function given in Theorem 4.2 , then there exists $c$ such that

$$
w_{\lambda}\left(x+c_{1}\right) \leqslant \lim _{t \rightarrow \infty} \inf u(x, t) \leqslant \lim _{t \rightarrow \infty} \sup u(x, t) \leqslant w_{\lambda}(x+c) \quad x \in R^{1} .
$$

\section{A problem with higher space dimension}

Theorem 5.1. Suppose that $\omega\left(x_{1}\right)$ satisfies

$$
\begin{aligned}
& \left\{\begin{array}{l}
\omega\left(x_{1}\right) \in \mathscr{B}^{2}(-\infty, 0], \\
\omega(0)=1, \quad \omega(-\infty)=0, \quad \omega^{\prime}\left(x_{1}\right) \geqslant 0 \quad x_{1} \leqslant 0,
\end{array}\right. \\
& \tilde{\omega}\left(x_{1}\right)=-\omega^{\prime \prime}\left(x_{1}\right)+2 \lambda \omega^{\prime}\left(x_{1}\right)-\lambda_{0}^{2} \omega\left(x_{1}\right) \geqslant 0 \quad x_{1} \leqslant 0, \\
& \lim _{x_{1} \rightarrow-\infty} \inf \frac{\omega\left(x_{1}\right)}{w_{\lambda}\left(x_{1}\right)}=0 .
\end{aligned}
$$

Suppose that the continuous function $u_{0}(x)$ defined on $n$-dimensional Euclidean space $R^{n}$ satisfies

$$
0 \leqslant u_{0}(x) \leqslant \begin{cases}1 & |x| \leqslant R, \\ \omega(R-|x|) & |x| \geqslant R\end{cases}
$$

for some $R \geqslant 0$. Then the solution $u(x, t)$ of the problem

$$
\left\{\begin{array}{l}
{\left[\frac{\partial}{\partial t}-\Delta\right] u=f(u), \quad 0 \leqslant u \leqslant 1 \quad(x, t) \in R^{n} \times(0, \infty),} \\
u(x, 0)=u_{0}(x) \quad x \in R^{n}
\end{array}\right.
$$


satisfies

$$
\lim _{t \rightarrow \infty} \sup _{|x|>2 \lambda t-\Delta} u(x, t)=0
$$

for any $A$.

Note that all of $\omega\left(x_{1}\right)$ given by Examples $4.1 \sim 4.5$ satisfy (5.1) (5.3). Suppose that the initial function $u_{0}(x)$ of the solution $u(x, t)$ of $(5.5)$ satisfies

$$
\sup _{|x|=r} u_{0}(x)= \begin{cases}O\left(\frac{1}{e^{\sigma_{-} r} \log ^{[n]} r}\right) & \left(\lambda>\lambda_{0}\right), \\ O\left(\frac{r}{e^{\lambda_{0} r} \log ^{[n]} r}\right) & \left(\lambda=\lambda_{0}\right) \quad \text { as } r \rightarrow+\infty .\end{cases}
$$

Here

$$
\log { }^{[1]} r=\log r, \quad \log { }^{[j+1]} r=\log \left(\log { }^{[j]} r\right) \quad j=1,2,3, \cdots .
$$

Then we have (5.6).

Proof of Theorem 5.1. Theorem 4.1 implies that the solution $u\left(x_{1}, t\right)$ of the problem

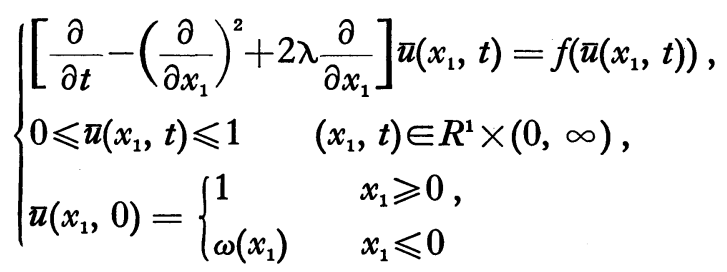

satisfies

$$
\lim _{t \rightarrow \infty} \sup _{x_{1}<A} \bar{u}\left(x_{1}, t\right)=0
$$

for any $A$. For any $\xi \in S^{n-1}=\left\{\xi \in R^{n} ;|\xi|=1\right\}$, we have

$$
\left\{\begin{array}{l}
{\left[\frac{\partial}{\partial t}-\Delta\right] u(\langle x, \xi\rangle+R+2 \lambda t, t)=f(\bar{u}(\langle x, \xi\rangle+R+2 \lambda t, t)),} \\
u(\langle x, \xi\rangle+R, 0)= \begin{cases}1 & \langle x, \xi\rangle+R \geqslant 0, \\
\omega(\langle x, \xi\rangle+R) & \langle x, \xi\rangle+R \leqslant 0 .\end{cases}
\end{array}\right.
$$

Here $\langle x, \xi\rangle=x_{1} \xi_{1}+\cdots+x_{n} \xi_{n}$ represents usual Euclidean inner product of $x=$ $\left(x_{1}, \cdots, x_{n}\right)$ and $\xi=\left(\xi_{1}, \cdots, \xi_{n}\right)$. Since $\omega^{\prime}\left(x_{1}\right) \geqslant 0 x_{1} \leqslant 0$, we have

$$
\omega(R-|x|) \leqslant \omega(\langle x, \xi\rangle+R) \quad\langle x, \xi\rangle+R \leqslant 0 .
$$

This implies

$$
0 \leqslant u_{0}(x) \leqslant u(\langle x, \xi\rangle+R, 0) \quad x \in R^{n}
$$


for any $\xi \in S^{n-1}$. By (5.5), (5.10) and (5.11) we have

$$
\begin{gathered}
0 \leqslant u(x, t) \leqslant u(\langle x, \xi\rangle+R+2 \lambda t, t) \\
(x, t) \in R^{n} \times[0, \infty), \quad \xi \in S^{n-1} .
\end{gathered}
$$

This gives

$$
\begin{aligned}
& \sup _{|x| \geqslant R+2 \lambda t-A} u(x, t)=\sup _{\xi \in S^{n-1}} \sup _{\langle x, \xi\rangle+R+2 \lambda t \leqslant A} u(x, t) \\
& \quad \leqslant \sup _{x_{1} \leqslant A} u\left(x_{1}, t\right) .
\end{aligned}
$$

(5.9) and (5.13) imply (5.6). This completes the proof of Theorem 5.1.

\section{Second stability theorem}

In this section we add the assumption:

$$
f^{\prime}(\xi) \leqslant f^{\prime}(0) \quad \xi \in[0,1] .
$$

Theorem 6.1. Suppose that $\vec{u}_{0}(x)$ and $\underline{u}_{0}(x)$ satisfies

$$
\begin{array}{ll}
0 \leqslant u_{0}(x) \leqslant w_{\lambda}(x) \leqslant u_{0}(x) \leqslant 1 & x \in R^{1}, \\
\int_{-\infty}^{0} e^{-\sigma_{-} x}\left\{u_{0}(x)-\underline{u}_{0}(x)\right\} d x & <+\infty .
\end{array}
$$

Suppose that the solution $u(x, t)$ of $(5)$ has the initial function $u_{0}(x) . \quad$ If

$$
\underline{u}_{0}(x) \leqslant u_{0}(x) \leqslant u_{0}(x) \quad x \in R^{1},
$$

then we have

$$
\lim _{t \rightarrow \infty} \sup _{x \leqslant A}\left|u(x, t)-w_{\lambda}(x)\right|=0
$$

for any $A$.

Proof. Let $\vec{u}(x, t)$ and $\underline{u}(x, t)$ be the solutions of the problem (5) with the initial function $\vec{u}_{0}(x)$ and $\underline{u}_{0}(x)$ respectively. On account of the comparison theorem to prove (6.5) it suffices to show that

$$
\lim _{t \rightarrow \infty} \sup _{x<A}\left\{u(x, t)-w_{\lambda}(x)\right\}=0
$$

and

$$
\lim _{t \rightarrow \infty} \sup _{x<A}\left\{w_{\lambda}(x)-\underline{u}(x, t)\right\}=0
$$

for any $A$. Put

$$
v(x, t)=\vec{u}(x, t)-w_{\lambda}(x) \quad\left(\text { or } w_{\lambda}(x)-\underline{u}(x, t)\right) .
$$

We have the differential inequality: 


$$
\left\{\begin{array}{l}
{\left[L+2 \lambda \frac{\partial}{\partial x}-\lambda_{0}^{2}\right] v \leqslant 0 \quad(x, t) \in R^{1} \times(0, \infty),} \\
0 \leqslant v(x, 0) \leqslant \bar{v}_{0}(x) \quad x \in R^{1} .
\end{array}\right.
$$

Here

$$
\bar{v}_{0}(x)= \begin{cases}1 & x \geqslant 0, \\ e^{\sigma_{-} x} k(x) & x \leqslant 0,\end{cases}
$$

$k(x)$ is a suitable continuous function which satisfies

$$
\int_{-\infty}^{0} k(y) d y=K<+\infty .
$$

Let $\bar{v}(x, t)$ be the solution of the problem

$$
\left\{\begin{array}{l}
{\left[L+2 \lambda \frac{\partial}{\partial x}-\lambda_{0}^{2}\right] \bar{v}=0 \quad(x, t) \in R^{1} \times(0, \infty),} \\
\bar{v}(x, 0)=\bar{v}_{0}(x) \quad x \in R^{1} .
\end{array}\right.
$$

By the comparison theorem we have

$$
0 \leqslant v(x, t) \leqslant \bar{v}(x, t) \quad(x, t) \in R^{1} \times[0, \infty) .
$$

(6.12) can be solved explicitly. We have

$$
\begin{aligned}
\bar{v}(x, t)= & \int_{-\infty}^{\infty} e^{\lambda(x-y)-\left(\lambda^{2}-\lambda_{0}^{2}\right) t} H(x-y, t) \bar{v}_{0}(y) d y \\
= & e^{\sigma_{-} x} \int_{-\infty}^{0} H\left(x-y-2 \sqrt{\lambda^{2}-\lambda_{0}^{2}} t, t\right) k(y) d y+ \\
& +\int_{0}^{\infty} e^{\lambda(x-y)-\left(\lambda^{2}-\lambda_{0}^{2}\right) t} H(x-y, t) d y .
\end{aligned}
$$

This gives

$$
\bar{v}(x, t) \leqslant \frac{1}{\sqrt{4 \pi t}}\left\{K e^{\sigma_{-} x}+\frac{1}{\lambda} e^{\lambda x-\left(\lambda^{2}-\lambda_{0}^{2}\right) t}\right\} .
$$

(6.13) and (6.15) prove (6.6) and (6.7). This completes the proof of Theorem 6.1.

\section{Part III. Method OF KPP}

\section{Fundamental theorem of KPP}

KPP has established an interesting result in [1]. Suppose that the KPP transform $\mathfrak{u}(\xi, \tau)$ of the solution $u(x, t)$ of (1) converges to some function as $\tau$ tends to infinity. Then $u(x, t)$ tends to one of the travelling waves $w_{\lambda}(x)$ in the manner which will be clarified soon after. The following theorem is a immediate generalization of this result. For the sake of completeness we give a detailed 
proof of it.

In this section $u(x, t)$ always represents the solution of (1) with $u_{0}(x) \in M$. $M$ is given by (7). We assume the existence of the limit:

$$
\lim _{\tau \rightarrow \infty} \hat{u}(\xi, \tau)=\hat{u}_{\infty}(\xi) \quad \xi \in(0,1)
$$

and supplementary condition:

$$
\hat{w}_{\lambda_{1}}(\xi) \leqslant \hat{u}(\xi, \tau) \leqslant \hat{W}(\xi) \quad(\xi, \tau) \in(0,1) \times[0, \infty)
$$

for some $\lambda_{1} \geqslant \lambda_{0}$ and $W(x) \in M(W(0)=1 / 2)$. Conclusion is as follows:

Theorem 7.1. There exists $\lambda$ satisfying $\lambda \geqslant \lambda_{0}$ such that

$$
\begin{aligned}
& \lim _{\tau \rightarrow \infty} \|\left(\frac{\partial}{\partial x}\right)^{j}\left(\frac{\partial}{\partial t}\right)^{k} u\left(x+u^{-1}(\xi, \tau), \tau\right)- \\
& -(2 \lambda)^{k}\left(\frac{d}{d x}\right)^{j+k} w_{\lambda}\left(x+w_{\lambda}^{-1}(\xi)\right) \|_{L^{p}}=0
\end{aligned}
$$

for any integers $j \geqslant 0, k \geqslant 0$ and any $p$ satisfying $1 \leqslant p \leqslant+\infty$.

$$
\lim _{\tau \rightarrow \infty}\left(\frac{\partial}{\partial \xi}\right)^{j}\left(\frac{\partial}{\partial \tau}\right)^{k}\left\{\hat{u}(\xi, \tau)-\hat{w}_{\lambda}(\xi)\right\}=0
$$

for any integers $j \geqslant 0$ and $k \geqslant 0$.

$$
\begin{aligned}
& \lim _{\tau \rightarrow \infty}\left\{u^{-1}(\xi, \tau)-u^{-1}\left(\frac{1}{2}, \tau\right)-w_{\lambda}^{-1}(\xi)\right\}=0 \\
& \lim _{\tau \rightarrow \infty} \sup _{0 \leqslant t \leqslant r}\left|u^{-1}(\xi, t+\tau)-u^{-1}(\xi, \tau)+2 \lambda t\right|=0
\end{aligned}
$$

for any $T>0$.

$$
\begin{aligned}
& \lim _{\tau \rightarrow \infty} \frac{\partial}{\partial \tau} u^{-1}(\xi, \tau)=-2 \lambda \\
& \lim _{\tau \rightarrow \infty}\left(\frac{\partial}{\partial \xi}\right)^{j}\left\{\frac{\partial}{\partial \xi} u^{-1}(\xi, \tau)-\frac{1}{\hat{w}_{\lambda}(\xi)}\right\}=0
\end{aligned}
$$

for any integer $j \geqslant 0$.

$$
\lim _{\tau \rightarrow \infty}\left(\frac{\partial}{\partial \xi}\right)^{j}\left(\frac{\partial}{\partial \tau}\right)^{k} u^{-1}(\xi, \tau)=0
$$

for any integers $j \geqslant 1, k \geqslant 1$ or $j \geqslant 0, k \geqslant 2$.

Convergence with respect to $\xi$ is uniform in every closed subinteeval of $(0,1)$ in all

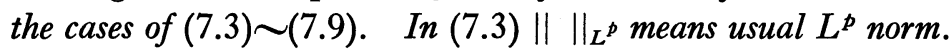

$$
\|u(x)\|_{L^{p}}= \begin{cases}\left\{\int_{-\infty}^{\infty}|u(x)|^{p} d x\right\}^{1 / p} & (1 \leqslant p<+\infty), \\ \sup _{x \in R^{1}}|u(x)| & (p=+\infty) .\end{cases}
$$


REMARK 7.1. If we have the additional condition:

$$
\dot{u}(\xi, \tau) \leqslant 0 \quad(\xi, \tau) \in(0,1) \times[0, \infty) .
$$

Then we have the monotonicity of the convergence in the following sense.

$$
(\operatorname{sen} x)\left\{u\left(x+u^{-1}(\xi, \tau), \tau\right)-w_{\lambda}\left(x+w_{\lambda}^{-1}(\xi)\right)\right\}(\nearrow 0
$$

as $\tau \nearrow+\infty$. Here $\operatorname{sgn} x=x /|x|$. As an immediate consequence of (7.3) we have

\section{REMARK 7.2.}

$$
\lim _{t \rightarrow \infty} \int_{-\infty}^{\infty}\left|u^{\prime}(x, t)\right|^{2} d x=\frac{1}{2 \lambda} \int_{0}^{1} f(\xi) d \xi
$$

Proof of Theorem 7.1.

First of all we have following relations:

$$
\begin{aligned}
& \left\{\begin{array}{rlrl}
W^{-1}(\xi) & =\int_{1 / 2}^{\xi} \frac{d \eta}{\hat{W}(\eta)} & \xi \in(0,1), \\
x & =\int_{1 / 2}^{W(x)} \frac{d \eta}{\hat{W}(\eta)} & & x \in R^{1},
\end{array}\right. \\
& \left\{\begin{aligned}
w_{\lambda}^{-1}(\xi) & =\int_{1 / 2}^{\xi} \frac{d \eta}{\hat{w}_{\lambda}(\eta)} & & \xi \in(0,1), \\
x & =\int_{1 / 2}^{w_{\lambda}(x)} \frac{d \eta}{\hat{w}_{\lambda}(\eta)} & & x \in R^{1},
\end{aligned}\right. \\
& \left\{\begin{aligned}
u_{\tau}^{-1}(\xi)=\int_{1 / 2}^{\xi} \frac{d \eta}{\hat{u}(\eta, \tau)} & \xi \in(0,1), \tau \geqslant 0, \\
x=\int_{1 / 2}^{u_{\tau}(x)} \frac{d \eta}{\hat{u}(\eta, \tau)} & x \in R^{1}, \tau \geqslant 0,
\end{aligned}\right. \\
& \left\{\begin{aligned}
u_{\infty}^{-1}(\xi) & =\int_{1 / 2}^{\xi} \frac{d \eta}{\hat{u}_{\infty}(\eta)} & & \xi \in(0,1), \\
x & =\int_{1 / 2}^{u_{\infty}(x)} \frac{d \eta}{\hat{u}_{\infty}(\eta)} & & x \in R^{1} .
\end{aligned}\right.
\end{aligned}
$$

Here $u_{\tau}^{-1}(\xi), u_{\tau}(x)$ and $u_{\infty}{ }^{-1}(\xi), u_{\infty}(x)$ are defined by the relations (7.15) and (7.16) respectively. It is easy to see

$$
\begin{aligned}
& u_{\tau}^{-1}(\xi)=u^{-1}(\xi, \tau)-u^{-1}\left(\frac{1}{2}, \tau\right), \\
& u_{\tau}(x)=u\left(x+u^{-1}\left(\frac{1}{2}, \tau\right), \tau\right) .
\end{aligned}
$$


It follows from (7.2) that

$$
\begin{aligned}
& \begin{cases}W^{-1}(\xi) \leqslant u_{\tau}^{-1}(\xi) \leqslant w_{\lambda_{1}}{ }^{-1}(\xi) & \frac{1}{2} \leqslant \xi \leqslant 1, \\
W^{-1}(\xi) \geqslant u_{\tau}^{-1}(\xi) \geqslant w_{\lambda_{1}}{ }^{-1}(\xi) & 0 \leqslant \xi \leqslant \frac{1}{2},\end{cases} \\
& \begin{cases}W(x) \geqslant u_{\tau}(x) \geqslant w_{\lambda_{1}}(x) & x \geqslant 0, \\
W(x) \leqslant u_{\tau}(x) \leqslant w_{\lambda_{1}}(x) & x \leqslant 0 .\end{cases}
\end{aligned}
$$

By (7.15) and (7.16) we have

$$
\begin{aligned}
& \int_{u_{\infty}(x)}^{u_{\tau}(x)} \frac{d \eta}{\hat{u}(\eta, \tau)}=x-u_{\tau}^{-1}\left(u_{\infty}(x)\right)= \\
= & \int_{1 / 2}^{u_{\infty}(x)}\left\{\frac{1}{\hat{u}_{\infty}(\eta)}-\frac{1}{\hat{u}(\eta, \tau)}\right\} d \eta .
\end{aligned}
$$

Since we have (7.1) and (7.2) this implies

$$
\lim _{\tau \rightarrow \infty} \sup _{|x| \leqslant A}\left|u_{\tau}(x)-u_{\infty}(x)\right|=0
$$

for any $A>0$. On the other hand (7.20) gives

$$
\left|u_{\tau}(x)-u_{\infty}(x)\right| \leqslant \begin{cases}1-w_{\lambda_{1}}(x) & x \geqslant 0 \\ w_{\lambda_{1}}(x) & x \leqslant 0\end{cases}
$$

Thus we obtain

\section{Lemma 7.1.}

$$
\lim _{\tau \rightarrow \infty}\left\|u_{\tau}(x)-u_{\infty}(x)\right\|_{L p}=0
$$

for any $p$ satisfying $1 \leqslant p \leqslant+\infty$.

Put

$$
u_{\tau}(x, t)=u\left(x+u^{-1}\left(\frac{1}{2}, \tau\right), t+\tau\right) .
$$

This is the solution of (1) with the initial function $u_{\tau}(x)$. Let $u_{\infty}(x, t)$ be the solution of (1) with the initial function $u_{\infty}(x)$.

In general the solution $v(x, t)$ of

$$
L v=f(x, t)
$$

has the representation:

$$
v(x, t)=\int_{-\infty}^{\infty} H\left(x-y, t-t_{0}\right) v\left(y, t_{0}\right) d y+
$$




$$
+\int_{t_{0}}^{t} \int_{-\infty}^{\infty} H(x-y, t-s) f(y, s) d y d s \quad(x, t) \in R^{1} \times\left[t_{0}, \infty\right) .
$$

Differentiating with respect to $x$, we have

$$
\begin{aligned}
& v^{\prime}(x, t)=\int_{-\infty}^{\infty}-\frac{x-y}{2\left(t-t_{0}\right)} H\left(x-y, t-t_{0}\right) v\left(y, t_{0}\right) d y+ \\
& +\int_{t_{0}}^{t} \int_{-\infty}^{\infty}-\frac{x-y}{2(t-s)} H(x-y, t-s) f(y, s) d y d s .
\end{aligned}
$$

Since

$$
\int_{-\infty}^{\infty} \frac{|x|}{2 t} H(x, t) d x=\frac{1}{\sqrt{\pi t}}
$$

we have

$$
\begin{aligned}
& \sup _{t_{1}<t<t_{2}}\left\|v^{\prime}(x, t)\right\|_{L p} \leqslant \frac{1}{\sqrt{\pi\left(t_{1}-t_{0}\right)}}\left\|v\left(x, t_{0}\right)\right\|_{L_{p}}+ \\
& +2 \sqrt{\frac{t_{2}-t_{0}}{\pi}} \sup _{t_{0}<t<t_{2}}\|f(x, t)\|_{L p}
\end{aligned}
$$

for any triple $t_{2}>t_{1}>t_{0}>0$ and any p satisfying $1 \leqslant p \leqslant+\infty$. Repeated applications of the above estimate to

$$
L\left\{u_{\tau}(x, t)-u_{\infty}(x, t)\right\}=f\left(u_{\tau}(x, t)\right)-f\left(u_{\infty}(x, t)\right)
$$

and Lemma 7.1 yield

\section{Lemma 7.2.}

$$
\lim _{\tau \rightarrow \infty} \sup _{t_{0}<t<t_{1}}\left\|\left(\frac{\partial}{\partial x}\right)^{j}\left(\frac{\partial}{\partial t}\right)^{k}\left\{u_{\tau}(x, t)-u_{\infty}(x, t)\right\}\right\|_{L_{p}}=0
$$

for any couple $t_{1}>t_{0}>0$, any integers $j \geqslant 0, k \geqslant 0$ and any $p$ satisfying $1 \leqslant p \leqslant+\infty$. In case of $j=k=0$ we can choose $t_{0}=0$.

Now we define $\varphi_{\tau}(t)$ and $\varphi_{\infty}(t)$ by the implicit relations:

$$
\begin{array}{ll}
u_{\tau}\left(\varphi_{\tau}(t), t\right)=\frac{1}{2} & t \geqslant 0, \tau \geqslant 0, \\
u_{\infty}\left(\varphi_{\infty}(t), t\right)=\frac{1}{2} & t \geqslant 0 .
\end{array}
$$

It is easy to see

$$
\varphi_{\tau}(t)=u^{-1}\left(\frac{1}{2}, t+\tau\right)-u^{-1}\left(\frac{1}{2}, \tau\right) .
$$

Since

$$
\begin{aligned}
& u_{\tau}(x, t) \in C^{\infty}\left(R^{1} \times[0, \infty)\right), \\
& u_{\infty}(x, t) \in C^{\infty}\left(R^{1} \times(0, \infty)\right) \cap C^{0}\left(R^{1} \times[0, \infty)\right),
\end{aligned}
$$


it follows

$$
\varphi_{\tau}(t) \in C^{\infty}[0, \infty), \varphi_{\infty}(t) \in C^{\infty}(0, \infty) \cap C^{0}[0, \infty) .
$$

Especially $\varphi_{\infty}(t)$ is bounded on $[0, T]$ for any $T>0$. By (7.33) and (7.34) we have

$$
\begin{aligned}
\int_{\varphi_{\tau}(t)}^{\varphi_{\infty}(t)} u_{\infty}^{\prime}(y, t) d y & =u_{\infty}\left(\varphi_{\infty}(t), t\right)-u_{\infty}\left(\varphi_{\tau}(t), t\right)= \\
& =u_{\tau}\left(\varphi_{\tau}(t), t\right)-u_{\infty}\left(\varphi_{\tau}(t), t\right) .
\end{aligned}
$$

Since we have

$$
u_{\infty}^{\prime}(x, t)>0 \quad(x, t) \in R^{1} \times[0, \infty)
$$

and

$$
\lim _{\tau \rightarrow \infty} \sup _{0 \leqslant t \leqslant T}\left\|u_{\tau}(x, t)-u_{\infty}(x, t)\right\|_{L^{\infty}}=0
$$

for any $T>0$. (7.36) gives

\section{Lemma 7.3.}

$$
\lim _{\tau \rightarrow \infty} \sup _{0<t \leqslant T}\left|\varphi_{\tau}(t)-\varphi_{\infty}(t)\right|=0
$$

for any $T>0$.

Differentiating (7.33) and (7.34) with respect to $t$ we have

$$
\begin{aligned}
& \dot{\varphi}_{\tau}(t)=-\frac{\dot{u}_{\tau}\left(\varphi_{\tau}(t), t\right)}{u_{\tau}^{\prime}\left(\varphi_{\tau}(t), t\right)}, \\
& \dot{\varphi}_{\infty}(t)=-\frac{\dot{u}_{\infty}\left(\varphi_{\infty}(t), t\right)}{u_{\infty}{ }^{\prime}\left(\varphi_{\infty}(t), t\right)} .
\end{aligned}
$$

Subtracting (7.40) from (7.41) we have

$$
\dot{\varphi}_{\infty}(t)-\dot{\varphi}_{\tau}(t)=\frac{I_{1}+I_{2}+I_{3}+I_{4}}{I_{0}} .
$$

Here

$$
\begin{aligned}
& I_{0}=u_{\tau}{ }^{\prime}\left(\varphi_{\tau}(t), t\right) u_{\infty}{ }^{\prime}\left(\varphi_{\infty}(t), t\right), \\
& I_{1}=\left\{\dot{u}_{\tau}\left(\varphi_{\tau}(t), t\right)-\dot{u}_{\infty}\left(\varphi_{\tau}(t), t\right)\right\} u_{\infty}{ }^{\prime}\left(\varphi_{\infty}(t), t\right), \\
& I_{2}=\left\{\dot{u}_{\infty}\left(\varphi_{\tau}(t), t\right)-\dot{u}_{\infty}\left(\varphi_{\infty}(t), t\right)\right\} u_{\infty}{ }^{\prime}\left(\varphi_{\infty}(t), t\right), \\
& I_{3}=\dot{u}_{\infty}\left(\varphi_{\infty}(t), t\right)\left\{u_{\infty}{ }^{\prime}\left(\varphi_{\infty}(t), t\right)-u_{\infty}{ }^{\prime}\left(\varphi_{\tau}(t), t\right)\right\}, \\
& I_{4}=\dot{u}_{\infty}\left(\varphi_{\infty}(t), t\right)\left\{u_{\infty}{ }^{\prime}\left(\varphi_{\tau}(t), t\right)-u_{\tau}{ }^{\prime}\left(\varphi_{\tau}(t), t\right)\right\} .
\end{aligned}
$$

Let us fix any $T>0$. There exists $R>0$ such that

$$
\sup _{0 \leqslant t \leqslant T}\left|\varphi_{\infty}(t)\right|<R .
$$

By Lemma 7.3 there exists $\tau_{0}>0$ such that 


$$
\sup _{0 \leqslant t \leqslant T, \tau \geqslant \tau_{0}}\left|\varphi_{\tau}(t)\right|<\mathrm{R} .
$$

Therefore by Lemma 7.2 and (7.37) we have

$$
\inf _{t_{0} \leqslant t<T, \tau \geqslant \tau_{0}} I_{0}>0
$$

for any fixed $t_{0}>0$. On the other hand we have

$$
\begin{aligned}
& \left|I_{1}\right| \leqslant\left\|\dot{u}_{\tau}-\dot{u}_{\infty}\right\|_{L^{\infty}}\left\|u_{\infty}{ }^{\prime}\right\|_{L^{\infty}}, \\
& \left|I_{2}\right| \leqslant\left|\varphi_{\tau}(t)-\varphi_{\infty}(t)\right|\left\|\dot{u}_{\infty}{ }^{\prime}\right\|_{L^{\infty}}\left\|u_{\infty}{ }^{\prime}\right\|_{L^{\infty}}, \\
& \left|I_{3}\right| \leqslant\left\|\dot{u}_{\infty}\right\|_{L^{\infty}}\left\|u_{\infty}{ }^{\prime \prime}\right\|_{L^{\infty}}\left|\varphi_{\tau}(t)-\varphi_{\infty}(t)\right|, \\
& \left|I_{4}\right| \leqslant\left\|\dot{u}_{\infty}\right\|_{L^{\infty}}\left\|u_{\infty}{ }^{\prime}-u_{\tau}{ }^{\prime}\right\|_{L^{\infty}} .
\end{aligned}
$$

Thus Lemmas 7.2 and 7.3 give

\section{Lemma 7.4.}

$$
\lim _{\tau \rightarrow \infty} \sup _{t_{0} \leqslant t \leqslant T}\left|\dot{\varphi}_{\tau}(t)-\dot{\varphi}_{\infty}(t)\right|=0
$$

for any couple $T>t_{0}>0$.

Put

$$
u_{\tau}\left(x+\varphi_{\tau}(t), t\right)-u_{\infty}\left(x+\varphi_{\infty}(t), t\right)=I_{1}+I_{2} .
$$

Here

$$
\begin{aligned}
& I_{1}=u_{\tau}\left(x+\varphi_{\tau}(t), t\right)-u_{\infty}\left(x+\varphi_{\tau}(t), t\right), \\
& I_{2}=u_{\infty}\left(x+\varphi_{\tau}(t), t\right)-u_{\infty}\left(x+\varphi_{\infty}(t), t\right) .
\end{aligned}
$$

Since

$$
\begin{aligned}
& \left\|I_{1}\right\|_{L^{\infty}}=\left\|u_{\tau}(x, t)-u_{\infty}(x, t)\right\|_{L^{\infty}}, \\
& \left\|I_{2}\right\|_{L^{\infty}} \leqslant\left|\varphi_{\tau}(t)-\varphi_{\infty}(t)\right|\left\|u_{\infty}^{\prime}(x, t)\right\|_{L^{\infty}},
\end{aligned}
$$

it follows from Lemmas 7.2 and 7.3 that

$$
\lim _{\tau \rightarrow \infty} \sup _{0 \leqslant t \leqslant T}\left\|u_{\tau}\left(x+\varphi_{\tau}(t), t\right)-u_{\infty}\left(x+\varphi_{\infty}(t), t\right)\right\|_{L^{\infty}}=0 .
$$

On the other hand we have

$$
u_{\tau}\left(x+\varphi_{\tau}(t), t\right)=u_{t+\tau}(x) .
$$

By Lemma 7.1 this shows

$$
\lim _{\tau \rightarrow \infty}\left\|u_{\tau}\left(x+\varphi_{\tau}(t), t\right)-u_{\infty}(x)\right\|_{L^{\infty}}=0
$$

for any $t \geqslant 0$. (7.50) and (7.52) shows 


\section{Lemma 7.5.}

$$
u_{\infty}\left(x+\varphi_{\infty}(t), t\right)=u_{\infty}(x) \quad(x, t) \in R^{1} \times[0 \infty,) .
$$

This implies $u_{\infty}(x) \in C^{\infty}\left(R^{1}\right)$. Differentiating (7.53) with respect to $t$, we have

$$
0=\dot{\varphi}_{\infty}(t) u_{\infty}^{\prime}\left(x+\varphi_{\infty}(t), t\right)+\dot{u}_{\infty}\left(x+\varphi_{\infty}(t), t\right) .
$$

Since $\dot{u}_{\infty}=u_{\infty}{ }^{\prime \prime}+f\left(u_{\infty}\right),(7.54)$ implies

$$
u_{\infty}^{\prime \prime}(x)+\dot{\varphi}_{\infty}(t) u_{\infty}{ }^{\prime}(x)+f\left(u_{\infty}(x)\right)=0 \quad x \in R^{1} .
$$

This shows that $\dot{\varphi}_{\infty}(t)$ is independent of $t$. Since $u_{\infty}(x) \in M$ we have

\section{Lemma 7.6.}

$$
\begin{aligned}
& \dot{\varphi}_{\infty}(t)=-2 \lambda \quad \text { for some } \lambda \geqslant \lambda_{0}, \\
& u_{\infty}(x)=w_{\lambda}(x) \quad x \in R^{1}, \\
& a_{\infty}(\xi)=\hat{w}_{\lambda}(\xi) \quad \xi \in(0,1), \\
& \lim _{\tau \rightarrow \infty} \hat{a}(\xi, \tau)=\hat{w}_{\lambda}(\xi) \quad \xi \in(0,1) .
\end{aligned}
$$

By (7.14) and (7.15) this gives

$$
\lim _{\tau \rightarrow \infty}\left\{u_{\tau}^{-1}(\xi)-w_{\lambda}^{-1}(\xi)\right\}=0
$$

uniformly with respect to $\xi$ in every closed subinterval of $(0,1)$, This proves (7.5). Since (7.57) gives

$$
u_{\infty}(x, t)=w_{\lambda}(x+2 \lambda t),
$$

it follows from Lemma 7.2 that

$$
\begin{aligned}
& \lim _{\tau \rightarrow \infty} \sup _{t_{0}<t<t_{1}} \|\left(\frac{\partial}{\partial x}\right)^{j}\left(\frac{\partial}{\partial t}\right)^{k} u\left(x+u^{-1}\left(\frac{1}{2}, \tau\right), t+\tau\right)- \\
& -(2 \lambda)^{k}\left(\frac{d}{d x}\right)^{j+k} w_{\lambda}(x+2 \lambda t) \|_{L^{p}}=0
\end{aligned}
$$

for any couple $t_{1}>t_{0}>0$, any integers $j \geqslant 0, k \geqslant 0$ and any $p$ satisfying $1 \leqslant p \leqslant+\infty$. Since $\varphi_{\infty}(0)=0,(7.56)$ gives

$$
\varphi_{\infty}(t)=-2 \lambda t
$$

Put

$$
\left(\frac{\partial}{\partial x}\right)^{j}\left(\frac{\partial}{\partial t}\right)^{k} u\left(x+u^{-1}\left(\frac{1}{2}, \tau+1\right), \tau+1\right)-
$$




$$
-(2 \lambda)^{k}\left(\frac{d}{d x}\right)^{j+k} w_{\lambda}(x)=I_{1}+I_{2}
$$

Here

$$
\begin{aligned}
I_{1}= & \left(\frac{\partial}{\partial x}\right)^{j}\left(\frac{\partial}{\partial t}\right)^{k} u\left(x+\varphi_{\tau}(1)+u^{-1}\left(\frac{1}{2}, \tau\right), \tau+1\right)- \\
& -(2 \lambda)^{k}\left(\frac{d}{d x}\right)^{j+k} w_{\lambda}\left(x+\varphi_{\tau}(1)+2 \lambda\right), \\
I_{2}= & (2 \lambda)^{k}\left(\frac{d}{d x}\right)^{j+k}\left\{w_{\lambda}\left(x+\varphi_{\tau}(1)-\varphi_{\infty}(1)\right)-w_{\lambda}(x)\right\} .
\end{aligned}
$$

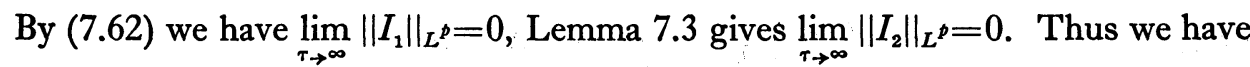

$$
\begin{gathered}
\lim _{\tau \rightarrow \infty} \|\left(\frac{\partial}{\partial x}\right)^{j}\left(\frac{\partial}{\partial t}\right)^{k} u\left(x+u^{-1}\left(\frac{1}{2}, \tau\right), \tau\right)- \\
-(2 \lambda)^{k}\left(\frac{d}{d x}\right)^{j+k} w_{\lambda}(x) \|_{L^{p}}=0
\end{gathered}
$$

for any integers $j \geqslant 0, k \geqslant 0$ and any $p$ satisfying $1 \leqslant p \leqslant+\infty$. Put

$$
\begin{aligned}
& \left(\frac{\partial}{\partial x}\right)^{j}\left(\frac{\partial}{\partial t}\right)^{k} u\left(x+u^{-1}(\xi, \tau), \tau\right)- \\
& \quad-(2 \lambda)^{k}\left(\frac{d}{d x}\right)^{j+k} w_{\lambda}\left(x+w_{\lambda}^{-1}(\xi)\right)=I_{1}+I_{2} .
\end{aligned}
$$

Here

$$
\begin{aligned}
I_{1}= & \left(\frac{\partial}{\partial x}\right)^{j}\left(\frac{\partial}{\partial t}\right)^{k} u\left(x+u_{\tau}^{-1}(\xi)+u^{-1}\left(\frac{1}{2}, \tau\right), \tau\right)- \\
& -(2 \lambda)^{k}\left(\frac{d}{d x}\right)^{j+k} w_{\lambda}\left(x+u_{\tau}^{-1}(\xi)\right), \\
I_{2}= & (2 \lambda)^{k}\left(\frac{d}{d x}\right)^{j+k}\left\{w_{\lambda}\left(x+u_{\tau}^{-1}(\xi)\right)-w_{\lambda}\left(x+w_{\lambda}^{-1}(\xi)\right)\right\} .
\end{aligned}
$$

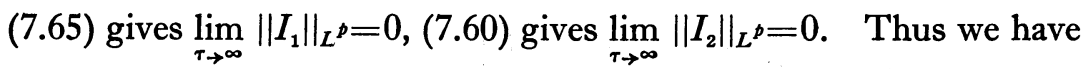

$$
\begin{aligned}
\lim _{\tau \rightarrow \infty} \|( & \left.\frac{\partial}{\partial x}\right)^{j}\left(\frac{\partial}{\partial t}\right)^{k} u\left(x+u^{-1}(\xi, \tau), \tau\right)- \\
& -(2 \lambda)^{k}\left(\frac{d}{d x}\right)^{j+k} w_{\lambda}\left(x+w_{\lambda}^{-1}(\xi)\right) \|_{L^{p}}=0
\end{aligned}
$$

for any integers $j \geqslant 0, k \geqslant 0$ and any $p$ satisfying $1 \leqslant p \leqslant+\infty$. This proves (7.3). Since

$$
\left|u^{\prime}\left(u^{-1}(\xi, \tau), \tau\right)-w_{\lambda}{ }^{\prime}\left(w_{\lambda}^{-1}(\xi)\right)\right| \leqslant\left\|u^{\prime}\left(x+u^{-1}(\xi, \tau), \tau\right)-w_{\lambda}{ }^{\prime}\left(x+w_{\lambda}{ }^{-1}(\xi)\right)\right\|_{L^{\infty}},
$$


(7.2) and (7.3) give

$$
\lim _{\tau \rightarrow \infty} \sup _{0<\xi<1}\left|\hat{a}(\xi, \tau)-\hat{w}_{\lambda}(\xi)\right|=0 .
$$

Since

$$
\begin{aligned}
& \left(\frac{\partial}{\partial \xi}\right)^{j} a(\xi, \tau)=\left.\left\{\frac{1}{u^{\prime}(x, t)} \frac{\partial}{\partial x}\right\}^{j} u^{\prime}(x, t)\right|_{x=u^{-1}(\xi, \tau), t=\tau}, \\
& \left(\frac{\partial}{\partial \xi}\right)^{j} \hat{w}_{\lambda}(\xi)=\left.\left\{\frac{1}{w_{\lambda}^{\prime}(x)} \frac{\partial}{\partial x}\right\}^{j} w_{\lambda}^{\prime}(x)\right|_{x=w_{\lambda}}{ }^{-1}(\xi),
\end{aligned}
$$

(7.3) gives

$$
\lim _{\tau \rightarrow \infty}\left(\frac{\partial}{\partial \xi}\right)^{j}\left\{\hat{u}(\xi, \tau)-\hat{w}_{\lambda}(\xi)\right\}=0
$$

for any integer $j \geqslant 0$. Since

$$
\begin{aligned}
& \dot{\boldsymbol{u}}=\hat{\mathfrak{a}}^{2} \hat{\boldsymbol{u}}^{\prime \prime}-f(\xi) \hat{u}^{\prime}+f^{\prime}(\xi) \hat{u}, \\
& 0=\hat{w}_{\lambda}{ }^{2} \hat{w}_{\lambda}{ }^{\prime \prime}-f(\xi) \hat{w}_{\lambda}{ }^{\prime}+f^{\prime}(\xi) \hat{w}_{\lambda},
\end{aligned}
$$

(7.69) gives

$$
\lim _{\tau \rightarrow \infty}\left(\frac{\partial}{\partial \xi}\right)^{j} \grave{u}(\xi, \tau)=0
$$

for any integer $j \geqslant 0$. If we assume

$$
\lim _{\tau \rightarrow \infty}\left(\frac{\partial}{\partial \xi}\right)^{j}\left(\frac{\partial}{\partial \tau}\right)^{l} \hat{u}(\xi, \tau)=0
$$

for any integers $j \geqslant 0$ and $l=1,2, \cdots, k-1$, then it follows from (19) that

$$
\lim _{\tau \rightarrow \infty}\left(\frac{\partial}{\partial \xi}\right)^{j}\left(\frac{\partial}{\partial \tau}\right)^{k} \mathfrak{a}(\xi, \tau)=0
$$

for any integer $j \geqslant 0$. This proves (7.4). (7.6) follows from (7.5) and Lemma 7.3. Since

$$
\begin{aligned}
& \frac{\partial}{\partial \tau} u^{-1}(\xi, \tau)=-\left\{\hat{a}^{\prime}+\frac{f(\xi)}{a}\right\}, \\
& \hat{w}_{\lambda}^{\prime}+\frac{f(\xi)}{\hat{w}_{\lambda}}=2 \lambda,
\end{aligned}
$$

(7.4) gives (7.7). Since

$$
\frac{\partial}{\partial \xi} u^{-1}(\xi, \tau)=\frac{1}{a},
$$

(7.8) follows from (7.4). (7.9) follows from (7.4) and (11) . This completes the 
proof of Theorem 7.1.

We shall next prove remark 7.1 and 7.2. (7.15) gives

$$
\begin{aligned}
& x+u^{-1}(\xi, \tau)-u^{-1}\left(\frac{1}{2}, \tau\right)=\int_{1 / 2}^{u\left(x+u^{-1}(\xi, \tau), \tau\right)} \frac{d \eta}{u(\eta, \tau)}, \\
& u^{-1}(\xi, \tau)-u^{-1}\left(\frac{1}{2}, \tau\right)=\int_{1 / 2}^{\xi} \frac{d \eta}{a(\eta, \tau)} .
\end{aligned}
$$

Subtracting (7.74) from (7.73) we get

$$
x=\int_{\xi}^{u\left(x+u^{-1}(\xi, \tau), \tau\right)} \frac{d \eta}{a(\eta, \tau)} .
$$

Differentiating with respect to $\tau$ we have

$$
\frac{\frac{d}{d \tau}\left\{\left\{u\left(x+u^{-1}(\xi, \tau), \tau\right)\right\}\right.}{a\left(u\left(x+u^{-1}(\xi, \tau), \tau\right), \tau\right)}=\int_{\xi}^{u(x+u-1(\xi, \tau), \tau)} \frac{\dot{\vec{u}}(\eta, \tau)}{\mathfrak{u}^{2}(\eta, \tau)} d \eta .
$$

This proves (7.11). (7.3) gives

$$
\lim _{t \rightarrow \infty} \int_{-\infty}^{\infty}\left|u^{\prime}(x, t)\right|^{2} d x=\int_{-\infty}^{\infty}\left|w_{\lambda}^{\prime}(x)\right|^{2} d x=\int_{0}^{1} \hat{w}_{\lambda}(\xi) d \xi .
$$

Since we have (2.12) this proves (7.12).

\section{Stability of the slowest travelling wave}

First we introduce the function $E(x)$ by

$$
E(x)=\int_{-\infty}^{x} H(y, 1) d y=\frac{1}{\sqrt{ } \pi} \operatorname{Erfc}\left(-\frac{x}{2}\right) .
$$

Here

$$
\operatorname{Erfc} x=\int_{x}^{\infty} e^{-y^{2}} d y
$$

is the error function of Gauss. It is easy to see

$$
\begin{aligned}
& E^{\prime}(x)=H(x, 1)>0 \quad x \in R^{1}, \\
& E(-\infty)=0, E(0)=\frac{1}{2}, E(+\infty)=1, \\
& E(x)+E(-x)=1 \quad x \in R^{1} .
\end{aligned}
$$

The well known asymptotic expansion

$$
x e^{x^{2}} \operatorname{Erfc} x \sim \sum_{n=0}^{\infty}(-1)^{n} \frac{(2 n-1) ! !}{2^{n+1}} x^{-2 n} \quad \text { as } x \rightarrow+\infty
$$

yields 


$$
\sqrt{\pi}|x| e^{x^{2} / 4} E(-|x|)=1+O\left(\frac{1}{|x|^{2}}\right) \quad \text { as } \quad|x| \rightarrow+\infty
$$

and

$$
C^{-1} \leqslant \frac{\left(\frac{|x|}{\sqrt{t}}+1\right) E\left(-\frac{|x|}{\sqrt{t}}\right)}{\sqrt{t} H(x, t)} \leqslant C \quad(x, t) \in R^{1} \times(0, \infty)
$$

for some $C>0$. The KPP transform $\hat{E}(\xi)$ of $E(x)$ satisfies

$$
\begin{aligned}
& \hat{E}(\xi)>0 \quad \xi \in(0,1) . \quad \hat{E}(0)=\hat{E}(1)=0 . \\
& \hat{E}(\xi)=\hat{E}(1-\xi) \quad \xi \in(0,1), \\
& \hat{E}^{\prime}(\xi)=-\frac{1}{2} E^{-1}(\xi) \quad \xi \in(0,1), \\
& \hat{E}(\xi) \hat{E}^{\prime \prime}(\xi)=-\frac{1}{2} \quad \xi \in(0,1), \\
& \lim _{\xi \rightarrow+0} \frac{\hat{E}(\xi)}{\xi \sqrt{-\log \xi}}=1, \\
& \lim _{\xi \rightarrow+0} \frac{\hat{E}^{\prime}(\xi)}{\sqrt{-\log \xi}}=1 .
\end{aligned}
$$

By (8.13) and (8.14) we can find $C>0$ such that

$$
\begin{array}{ll}
C^{-1} \leqslant \frac{\hat{E}(\xi)}{\xi(1-\xi) \sqrt{-\log \xi(1-\xi)}} \leqslant C & \xi \in(0,1), \\
\left|\hat{E}^{\prime}(\xi)\right| \leqslant C \sqrt{-\log \xi(1-\xi)} & \xi \in(0,1) .
\end{array}
$$

\section{Lemma 8.1. Put}

$$
a_{0}(\xi)=\frac{1}{\sqrt{\delta}} \hat{E}(\xi)
$$

and

$$
\frac{1}{\delta_{0}}=\max \left\{2 \sup _{0<\xi<1]}\left|f^{\prime}(\xi)-f(\xi) \frac{\hat{E}^{\prime}(\xi)}{\hat{E}(\xi)}\right|, \sup _{0<\xi<1}\left|\hat{w}_{\lambda_{0}}(\xi)\right|^{2}\right\} .
$$

For any $\delta$ satisfying $0<\delta \leqslant \delta_{0}$, we have

$$
\begin{aligned}
& \hat{u}_{0}(\xi) \geqslant \hat{w}_{\lambda_{0}}(\xi) \quad \xi \in(0,1), \\
& \hat{u}_{0}^{2}(\xi) \hat{u}_{0}{ }^{\prime \prime}(\xi)-f(\xi) \hat{u}_{0}{ }^{\prime}(\xi)+f^{\prime}(\xi) \hat{a}_{0}(\xi) \leqslant 0 \quad \xi \in(0,1) .
\end{aligned}
$$

The conclusions of this section is as follows

Theorem 8.1. Suppose that $u(x, t)$ is the solution of (1) with the initial function 


$$
u_{0}(x)=E\left(\frac{x}{\sqrt{\delta}}\right) \quad x \in R^{1}
$$

for some $\delta$ satisfying $0<\delta \leqslant \delta_{0}$. Here $\delta_{0}$ is given by (8.18). Then we have

$$
\begin{aligned}
& \mathfrak{u}(\xi, \tau) \geqslant \hat{w}_{\lambda_{0}}(\xi) \quad(\xi, \tau) \in(0,1) \times[0, \infty), \\
& \grave{a}(\xi, \tau) \leqslant 0 \quad(\xi, \tau) \in(0,1) \times[0, \infty), \\
& \lim _{\tau \rightarrow \infty} \sup _{0<\xi<1}\left|\hat{a}(\xi, \tau)-\hat{w}_{\lambda_{0}}(\xi)\right|=0 .
\end{aligned}
$$

All the conclusions (7.3) (7.9) of Theorem 7.1 are valid replacing $\lambda$ by $\lambda_{0}$. Especially we have

$$
\begin{array}{r}
(\operatorname{sgn} x)\left\{u\left(x+u^{-1}(\xi, \tau), \tau\right)-w_{\lambda_{0}}\left(x+w_{\lambda_{0}}{ }^{-1}(\xi)\right)\right\} \searrow 0 \\
\text { as } \tau \nearrow+\infty .
\end{array}
$$

Theorem 8.2. Suppose that $u(x, t)$ and $u_{0}(x)$ are the same as above. Suppose that $v(x, t)$ is the solution of $(1)$ with the initial function $v_{0}(x) \in M$ satisfying

$$
\hat{w}_{\lambda_{0}}(\xi) \leqslant \hat{v}_{0}(\xi) \leqslant \hat{u}_{0}(\xi) \quad \xi \in(0,1) .
$$

Then we have

$$
\begin{aligned}
& \hat{w}_{\lambda_{0}}(\xi) \leqslant \hat{v}(\xi, \tau) \leqslant \hat{u}(\xi, \tau) \quad(\xi, \tau) \in(0,1) \times[0, \infty), \\
& \lim _{\tau \rightarrow \infty} \sup _{0<\xi<1}\left|\hat{v}(\xi, \tau)-\hat{w}_{\lambda_{0}}(\xi)\right|=0 .
\end{aligned}
$$

All the conclusions (7.3) (7.9) of Theorem 7.1 are valid replacing $u(x, t)$ and $\lambda$ by $v(x, t)$ and $\lambda_{0}$ respectively.

Proof of Theorem 8.1.

To prove Theorem 8.1 it is sufficient to show (8.22) and (8.23). The remaining parts of the conclusions follow from Theorem 7.1. Formally (8.22) and (8.23) follow from (25) and (28) respectively. All we have to do is to show the applicability of the comparison theorem to (25) and (28).

Lemma 8.2. The following inequalities hold on $R^{1} \times[0, \infty)$ with suitable $A \geqslant 0$ and $B \geqslant 0$.

$$
\begin{aligned}
& E\left(\frac{x}{\sqrt{t+\delta}}\right) \leqslant u(x, t) \leqslant e^{A t} E\left(\frac{x}{\sqrt{t+\delta}}\right), \\
& u(x, t)\{1-u(x, t)\} \leqslant e^{A t} E\left(-\frac{|x|}{\sqrt{\overline{t+\delta}}}\right), \\
& e^{-A t} H(x, t+\delta) \leqslant u^{\prime}(x, t) \leqslant e^{A t} H(x, t+\delta),
\end{aligned}
$$




$$
\begin{aligned}
& \left|u^{\prime \prime}(x, t)\right| \leqslant e^{A t+B}\left\{\frac{x^{2}}{t+\delta}+1\right\}^{1 / 2} H(x, t+\delta), \\
& \left|u^{\prime \prime \prime}(x, t)\right| \leqslant e^{A t+B}\left\{\frac{x^{2}}{t+\delta}+1\right\} H(x, t+\delta) .
\end{aligned}
$$

Proof. Note that we have the following relations:

$$
\begin{aligned}
& \left\{\begin{array}{l}
L u=f(u), \\
u(x, 0)=E\left(\frac{x}{\sqrt{\delta}}\right),
\end{array}\right. \\
& \left\{\begin{array}{l}
{\left[L-f^{\prime}(u)\right] u^{\prime}=0,} \\
u^{\prime}(x, 0)=H(x, \delta),
\end{array}\right. \\
& \left\{\begin{array}{l}
{\left[L-f^{\prime}(u)\right] u^{\prime \prime}=f^{\prime \prime}(u)\left(u^{\prime}\right)^{2},} \\
u^{\prime \prime}(x, 0)=-\frac{x}{2 \delta} H(x, \delta),
\end{array}\right. \\
& \left\{\begin{array}{l}
{\left[L-f^{\prime}(u)\right] u^{\prime \prime \prime}=3 f^{\prime \prime}(u) u^{\prime} u^{\prime \prime}+f^{\prime \prime \prime}(u)\left(u^{\prime}\right)^{3},} \\
u^{\prime \prime \prime}(x, 0)=\left(\frac{x^{2}}{4 \delta^{2}}-\frac{1}{2 \delta}\right) H(x, \delta) .
\end{array}\right.
\end{aligned}
$$

Since

$$
L E\left(\frac{x}{\sqrt{t+\delta}}\right)=0, L H(x, t+\delta)=0 \quad(x, t) \in R^{1} \times(0, \infty),
$$

it is easy to see (8.29) and (8.31). (8.30) follows from (8.29). Put

$$
h_{k}(x, t)=e^{A_{k} t+B_{k}}\left\{\frac{x^{2}}{t+\delta}+1\right\}^{k / 2} H(x, t+\delta) \quad k=1,2 .
$$

There exists $C_{k} \geqslant 0$, which is independent of the choice of $A_{k}$ and $B_{k}$, such that

$$
\left[L-f^{\prime}(u)\right] h_{k} \geqslant\left(A_{k}-C_{k}\right) h_{k} \quad(x, t) \in R^{1} \times(0, \infty) \quad k=1,2 .
$$

Taking $A_{1}$ and $B_{1}$ sufficiently large, we see that (8.31), (8.36) and (8.39) give

$$
\begin{cases}{\left[L-f^{\prime}(u)\right]\left(h_{1} \pm u^{\prime \prime}\right) \geqslant 0} & (x, t) \in R^{1} \times(0, \infty), \\ \left.\left(h_{1} \pm u^{\prime \prime}\right)\right|_{t=0} \geqslant 0 & x \in R^{1} .\end{cases}
$$

The estimate (30) assures the applicability of the comparison theorem to (8.40). Thus we have

$$
\left|u^{\prime \prime}(x, t)\right| \leqslant h_{1}(x, t) \quad(x, t) \in R^{1} \times[0, \infty) .
$$

This proves (8.32). Taking $A_{2}$ and $B_{2}$ sufficiently large, we can see that (8.31), 
(8.32), (8.37) and (8.39) give

$$
\begin{cases}{\left[L-f^{\prime}(u)\right]\left(h_{2} \pm u^{\prime \prime \prime}\right) \geqslant 0} & (x, t) \in R^{1} \times(0, \infty), \\ \left.\left(h_{2} \pm u^{\prime \prime \prime}\right)\right|_{t=0} \geqslant 0 & x \in R^{1} .\end{cases}
$$

The comparison theorem yields

$$
\left|u^{\prime \prime \prime}(x, t)\right| \leqslant h_{2}(x, t) \quad(x, t) \in R^{1} \times[0, \infty) .
$$

This proves (8.33). This completes the proof of Lemma 8.2. By (8.19) and (25) we have

$$
\left\{\begin{array}{l}
{\left[L-c_{1}(x, t)\right]\left(u^{\prime}-\hat{w}_{\lambda_{0}}(u)\right)=0} \\
\left.\left(u^{\prime}-\hat{w}_{\lambda_{0}}(u)\right)\right|_{t=0} \geqslant 0
\end{array}\right.
$$

Here

$$
c_{1}(x, t)=f^{\prime}(u(x, t))+\left\{u^{\prime}(x, t)+\hat{w}_{\lambda_{0}}(u(x, t))\right\} \hat{w}_{\lambda_{0}}^{\prime \prime}(u(x, t)) .
$$

Since $u \hat{w}_{\lambda_{n}}{ }^{\prime \prime}(u)$ is bounded, (8.29) and (8.31) give

$$
c_{1}(x, t) \leqslant e^{A t+B}\left(x^{2}+1\right)^{1 / 2} \quad(x, t) \in R^{1} \times[0, \infty)
$$

for some $A \geqslant 0$ and $B \geqslant 0$. This assures the applicability of the comparison theorem to (8.44). Thus we have

$$
u^{\prime}(x, t)-\hat{w}_{\lambda_{0}}(u(x, t)) \geqslant 0 \quad(x, t) \in R^{1} \times[0, \infty) .
$$

This proves (8.22). By (8.20) and (28) we have

$$
\left\{\begin{array}{l}
{\left[L-c_{2}(x, t)\right] \hat{u}(u(x, t), t)=0 \quad(x, t) \in R^{1} \times(0, \infty),} \\
\hat{u}(u(x, 0), 0) \leqslant 0 .
\end{array}\right.
$$

Here

$$
\begin{aligned}
& c_{2}(x, t)=f^{\prime}(u(x, t))+2\left\{\frac{u^{\prime \prime \prime}(x, t)}{u^{\prime}(x, t)}-\left(\frac{u^{\prime \prime}(x, t)}{u^{\prime}(x, t)}\right)^{2}\right\} \\
& \dot{u}(u(x, t), t)=u^{\prime \prime \prime}(x, t)-\frac{\left(u^{\prime \prime}(x, t)\right)^{2}}{u^{\prime}(x, t)}- \\
& -f(u(x, t)) \frac{u^{\prime \prime}(x, t)}{u^{\prime}(x, t)}+f^{\prime}(u(x, t)) u^{\prime}(x, t) .
\end{aligned}
$$

Lemma 8.2 gives

$$
\begin{array}{ll}
c_{2}(x, t) \leqslant e^{A t+B}\left(x^{2}+1\right) & (x, t) \in R^{1} \times[0, \infty), \\
|\dot{u}(u(x, t), t)| \leqslant e^{A t+B} & (x, t) \in R^{1} \times[0, \infty)
\end{array}
$$

for some $A \geqslant 0$ and $B \geqslant 0$. (8.51) and (8.52) assures the applicability of the 
comparison theorem to (8.48). Thus we have

$$
\dot{u}(u(x, t), t) \leqslant 0 \quad(x, t) \in R^{1} \times[0, \infty) .
$$

This proves (8.23). This completes the proof of Theorem 8.1.

Proof of Theorem 8.2.

To prove Theorem 8.2 it is sufficient to show (8.27). The remaining parts follow from Theorems 8.1 and 7.1.

\section{Lemma 8.3.}

$$
\hat{v}(\xi, \tau) \leqslant \hat{u}_{0}(\xi) \quad(\xi, \tau) \in(0,1) \times[0, \infty) .
$$

Proof. (8.20) gives

$$
\left[\hat{L}_{\hat{u}_{0}}-f^{\prime}(\xi)\right] \hat{u}_{0}(\xi) \geqslant 0 \quad \xi \in(0,1) .
$$

Replacing $\hat{u}$ and $h$ by $\hat{v}$ and $\hat{u}_{0}$ in (29) we have

$$
\left\{\begin{array}{l}
{\left[\hat{L}_{\hat{v}}-\left\{f^{\prime}(\xi)+\left(\hat{v}+\hat{u}_{0}\right) \hat{u}_{0}{ }^{\prime \prime}\right\}\right]\left(\hat{u}_{0}-\hat{v}\right) \geqslant 0,} \\
\left.\left(\hat{u}_{0}-\hat{v}\right)\right|_{\tau=0} \geqslant 0 .
\end{array}\right.
$$

Since $\hat{v}>0, \hat{u}_{0}>0$ and $\hat{u}_{0}^{\prime \prime}<0$ this implies (8.54). This completes the proof of Lemma 8.3. Since

$$
\left[\hat{L}_{\hat{w}_{\lambda_{0}}}-f^{\prime}(\xi)\right] \hat{w}_{\lambda_{0}}(\xi)=0 \quad \xi \in(0,1) .
$$

Replacing $\boldsymbol{a}$ and $h$ by $\hat{v}$ and $\hat{w}_{\lambda_{0}}$ in (29) we have

$$
\left\{\begin{array}{l}
{\left[\hat{L}_{\hat{v}}-\left\{f^{\prime}(\xi)+\left(\hat{v}+\hat{w}_{\lambda_{0}}\right) \hat{w}_{\lambda_{0}}{ }^{\prime \prime}\right\}\right]\left(\hat{v}-\hat{w}_{\lambda_{0}}\right)=0,} \\
\left.\left(\hat{v}-\hat{w}_{\lambda_{0}}\right)\right|_{\tau=0} \geqslant 0 .
\end{array}\right.
$$

(1.79) and (8.13) show that $\hat{u}_{0}(\xi) \hat{w}_{\lambda_{0}}{ }^{\prime \prime}(\xi)$ and $\hat{w}_{\lambda_{0}}(\xi) \hat{w}_{\lambda_{0}}{ }^{\prime \prime}(\xi)$ are bounded. Therefore (8.54) shows that $\left\{f^{\prime}(\xi)+\left(\hat{v}+\hat{w}_{\lambda_{0}}\right) \hat{w}_{\lambda_{0}}{ }^{\prime \prime}\right\}$ is bounded. Thus (8.56) gives

$$
\hat{w}_{\lambda_{0}}(\xi) \leqslant \hat{v}(\xi, \tau) \quad(\xi, \tau) \in(0,1) \times[0, \infty) .
$$

\section{Lemma 8.4.}

$$
\hat{u}(\xi, \tau) \geqslant e^{-A^{\tau}} \hat{u}_{0}(\xi) \quad(\xi, \tau) \in(0,1) \times[0, \infty)
$$

for some $A \geqslant 0$.

Proof. Put

$$
h(\xi, \tau)=e^{-A \tau} \hat{u}_{0}(\xi) .
$$

Taking $A$ sufficiently large, we have 


$$
\left[\hat{L}_{h}-f^{\prime}(\xi)\right] h \leqslant 0 \quad(\xi, \tau) \in(0,1) \times[0, \infty) .
$$

Therefore (29) gives

$$
\left\{\begin{array}{l}
{\left[\hat{L}_{\hat{u}}-\left\{f^{\prime}(\xi)+(\hat{u}+h) h^{\prime \prime}\right\}\right](\hat{u}-h) \geqslant 0,} \\
\left.(\hat{u}-h)\right|_{\tau=0}=0 .
\end{array}\right.
$$

Since $\hat{u}>0, h>0$ and $h^{\prime \prime}<0,(8.60)$ gives (8.58). This completes the proof of Lemma 8.4. Replacing $\hat{u}$ and $h$ by $\hat{v}$ and $\hat{u}$ respectively in (29) we have

$$
\left\{\begin{array}{l}
{\left[\hat{L}_{\hat{v}}-\left\{f^{\prime}(\xi)+(\hat{v}+\hat{u}) \hat{u}^{\prime \prime}\right\}\right](\hat{u}-\hat{v})=0,} \\
\left.(\hat{u}-\hat{v})\right|_{\tau=0} \geqslant 0 .
\end{array}\right.
$$

Note that (8.54) and (8.58) give

$$
\hat{v}(\xi, \tau) \leqslant e^{A \tau} \hat{u}(\xi, \tau) \quad(\xi, \tau) \in(0,1) \times[0, \infty)
$$

for some $A \geqslant 0$. (19) and (8.23) give

$$
\hat{u} \hat{u}^{\prime \prime} \leqslant f(\xi) \frac{\hat{u}^{\prime}}{\hat{u}}-f^{\prime}(\xi) \quad(\xi, \tau) \in(0,1) \times[0, \infty) .
$$

Lemma 8.2 shows that

$$
f(\xi) \frac{\hat{u}^{\prime}}{\hat{u}} \leqslant e^{A \tau+B} \quad(\xi, \tau) \in(0,1) \times[0, \infty)
$$

for some $A \geqslant 0$ and $B \geqslant 0$. (8.62), (8.63), and (8.64) give

$$
\left\{f^{\prime}(\xi)+(\hat{v}+\hat{u}) \hat{u}^{\prime \prime}\right\} \leqslant e^{A \tau+B} \quad(\xi, \tau) \in(0,1) \times[0, \infty)
$$

for some $A \geqslant 0$ and $B \geqslant 0$. This assures the applicability of the comparison theorem to (8.61). It follows

$$
\hat{v}(\xi, \tau) \leqslant \hat{u}(\xi, \tau) \quad(\xi, \tau) \in(0,1) \times[0, \infty) .
$$

(8.57) and (8.66) prove (8.27). This completes the proof of Theorem 8.2.

\section{Stability of the travelling wave with arbitrary speed}

Suppose that the solution $u(x, t)$ of $(1)$ has the initial function $u_{0}(x)$. Throughout this section we assume that $u_{0}(x)$ belongs to the class $N$. Here $u_{0}(x)$ belongs to the class $N$, if and only if $u_{0}(x)$ belongs to the class $M$ and the KPP transform $\hat{u}_{0}(\xi)$ of $u_{0}(x)$ satisfies the following conditions:

$$
\begin{aligned}
& \hat{u}_{0}(\xi) \in C^{0}[0,1] \cap C^{2}(0,1), \\
& C^{-1} \leqslant \frac{\hat{u}_{0}(\xi)}{\xi(1-\xi)} \leqslant C \quad \xi \in(0,1)
\end{aligned}
$$


for some $C>0$.

$$
\hat{u}_{0}^{\prime}(\xi) \text { and } \hat{u}_{0}(\xi) \hat{u}_{0}^{\prime \prime}(\xi) \text { are bounded on }(0,1) \text {. }
$$

Put

$$
p(\xi)=c \xi(1-\xi) \quad \xi \in(0,1) .
$$

There exist $\lambda_{1} \geqslant \lambda_{0}$ and $c>0$ such that

$$
\begin{aligned}
& \hat{w}_{\lambda_{1}}(\xi) \leqslant \hat{u}_{0}(\xi) \leqslant p(\xi) \quad \xi \in(0,1), \\
& 0 \geqslant p^{2} p^{\prime \prime}-f(\xi) p^{\prime}+f^{\prime}(\xi) p \quad \xi \in(0,1) .
\end{aligned}
$$

Lemma 9.1. Following inequalities hold on $R^{1} \times[0, \infty)$ with suitable $A \geqslant 0$ and $B \geqslant 0$.

$$
\begin{aligned}
& \hat{w}_{\lambda_{1}}(u(x, t)) \leqslant u^{\prime}(x, t) \leqslant p(u(x, t)), \\
& \left|u^{\prime \prime}(x, t)\right| \leqslant e^{A t+B} p(u(x, t)), \\
& \left|u^{\prime \prime \prime}(x, t)\right| \leqslant e^{A t+B} p(u(x, t)) .
\end{aligned}
$$

Proof. Since

$$
\begin{aligned}
& \left\{\begin{array}{l}
{\left[\hat{L}_{\hat{u}}-\left\{f^{\prime}(\xi)+(\hat{u}+p) p^{\prime \prime}\right\}\right](p-\hat{u}) \geqslant 0,} \\
\left.(p-\hat{u})\right|_{\tau=0} \geqslant 0,
\end{array}\right. \\
& \left\{\begin{array}{l}
{\left[\hat{L}_{\hat{u}}-\left\{f^{\prime}(\xi)+\left(\hat{u}+\hat{w}_{\lambda_{1}}\right) \hat{w}_{\lambda_{1}}{ }^{\prime \prime}\right\}\right]\left(\hat{u}-\hat{w}_{\lambda_{1}}\right)=0,} \\
\left.\left(\hat{u}-\hat{w}_{\lambda_{1}}\right)\right|_{\tau=0} \geqslant 0,
\end{array}\right.
\end{aligned}
$$

it follows (9.7). Since

$$
\begin{aligned}
& u_{0}^{\prime \prime}(x)=\left.\hat{u}_{0}(\xi) \hat{u}_{0}{ }^{\prime}(\xi)\right|_{\xi=u_{0}(x)}, \\
& u_{0}^{\prime \prime \prime}(x)=\left.\hat{u}_{0}(\xi)\left\{\hat{u}_{0}(\xi) \hat{u}_{0}^{\prime \prime}(\xi)+\left(\hat{u}_{0}^{\prime}(\xi)\right)^{2}\right\}\right|_{\xi=u_{0}(x)},
\end{aligned}
$$

there exists $B \geqslant 0$ such that

$$
\begin{array}{ll}
\left|u_{0}^{\prime \prime}(x)\right| \leqslant e^{B} p\left(u_{0}(x)\right) & x \in R^{1}, \\
\left|u_{0}^{\prime \prime \prime}(x)\right| \leqslant e^{B} p\left(u_{0}(x)\right) & x \in R^{1} .
\end{array}
$$

Put

$$
h_{k}(x, t)=e^{A_{k} t+B_{k} p(u(x, t))} \quad k=1,2 .
$$

Since

$$
\left[L-f^{\prime}(u)\right] h_{k}=h_{k}(x, t)\left[A_{k}-f^{\prime}(\xi)-\frac{\hat{u}^{2} p^{\prime \prime}-f(\xi) p^{\prime}}{p}\right]_{\xi=u(x, t), \tau=t}
$$


There exists $C \geqslant 0$, which is independent of the choice of $A_{k}$ and $B_{k}$, such that

$$
\left[L-f^{\prime}(u)\right] h_{k} \geqslant\left(A_{k}-C\right) h_{k} \quad k=1,2 .
$$

On the other hand we have

$$
\begin{aligned}
& \left\{\begin{array}{l}
{\left[L-f^{\prime}(u)\right] u^{\prime \prime}=f^{\prime \prime}(u)\left(u^{\prime}\right)^{2},} \\
u^{\prime \prime}(x, 0)=u_{0}^{\prime \prime}(x),
\end{array}\right. \\
& \left\{\begin{array}{l}
{\left[L-f^{\prime}(u)\right] u^{\prime \prime \prime}=3 f^{\prime \prime}(u) u^{\prime} u^{\prime \prime}+f^{\prime \prime \prime}(u)\left(u^{\prime}\right)^{3},} \\
u^{\prime \prime \prime}(x, 0)=u_{0}^{\prime \prime \prime}(x) .
\end{array}\right.
\end{aligned}
$$

Taking $A_{1}$ and $B_{1}$ sufficiently large, it follows from (9.7), (9.14), (9.18) and (9.19) that

$$
\begin{cases}{\left[L-f^{\prime}(u)\right]\left(h_{1} \pm u^{\prime \prime}\right) \geqslant 0} & (x, t) \in R^{1} \times(0, \infty), \\ \left.\left(h_{1} \pm u^{\prime \prime}\right)\right|_{t=0} \geqslant 0 & x \in R^{1} .\end{cases}
$$

This gives

$$
\left|u^{\prime \prime}(x, t)\right| \leqslant h_{1}(x, t) \quad(x, t) \in R^{1} \times[0, \infty) .
$$

This proves (9.8). Taking $A_{2}$ and $B_{2}$ sufficiently large, it follows from (9.7), (9.8), (9.15), (9.18) and (9.20) that

$$
\begin{cases}{\left[L-f^{\prime}(u)\right]\left(h_{2} \pm u^{\prime \prime \prime}\right) \geqslant 0} & (x, t) \in R^{1} \times(0, \infty), \\ \left.\left(h_{2} \pm u^{\prime \prime \prime}\right)\right|_{t=0} \geqslant 0 & x \in R^{1}\end{cases}
$$

This gives

$$
\left|u^{\prime \prime \prime}(x, t)\right| \leqslant h_{2}(x, t) \quad(x, t) \in R^{1} \times(0, \infty) .
$$

This proves (9.9). This completes the proof of Lemma 9.1.

Lemma 9.2. If

$$
\left\{\hat{a}_{0}^{\prime}(\xi)+\frac{f(\xi)}{\hat{u}_{0}(\xi)}\right\}_{(\geqslant)}^{\prime} \underset{0}{\leqslant} \quad \xi \in(0,1)
$$

then we have

$$
\hat{u}(\xi, \tau) \underset{(\geqslant)}{\leqslant 0} \quad(\xi, \tau) \in(0,1) \times[0, \infty) .
$$

Proof. By (28) and (9.25) we have

$$
\begin{cases}{\left[L-c_{2}(x, t)\right] \hat{u}(u(x, t), t)=0} & (x, t) \in R^{1} \times(0, \infty), \\ \dot{u}(u(x, 0), 0) \leqslant 0 & x \in R^{1} .\end{cases}
$$


Here

$$
\begin{aligned}
& c_{2}(x, t)=f^{\prime}(u(x, t))+2\left\{\frac{u^{\prime \prime \prime}(x, t)}{u^{\prime}(x, t)}-\left(\frac{u^{\prime \prime}(x, t)}{u^{\prime}(x, t)}\right)^{2}\right\}, \\
& \hat{u}(u(x, t), t)=u^{\prime \prime \prime}(x, t)-\frac{\left(u^{\prime \prime}(x, t)\right)^{2}}{u^{\prime}(x, t)}- \\
& -f(u(x, t)) \frac{u^{\prime \prime}(x, t)}{u^{\prime}(x, t)}+f^{\prime}(u(x, t)) u^{\prime}(x, t) .
\end{aligned}
$$

Lemma 9.1 gives

$$
\begin{aligned}
& c_{2}(x, t) \leqslant e^{A t+B} \quad(x, t) \in R^{1} \times[0, \infty), \\
& |\hat{u}(u(x, t), t)| \leqslant e^{A t+B} p(u(x, t)) \quad(x, t) \in R^{1} \times[0, \infty)
\end{aligned}
$$

for some $A \geqslant 0$ and $B \geqslant 0$. Thus the comparison theorem can be applied to (9.27). (9.27) gives

$$
\dot{u}(u(x, t), t) \underset{(\geqslant)}{\leqslant 0} \quad(x, t) \in R^{1} \times[0, \infty) .
$$

This proves Lemma 9.2.

Theorem 9.1. Suppose that the solution $u(x, t)$ of (1) has the initial function $u_{0}(x)$ which belongs to the class $N$. Suppose that we have

$$
\begin{aligned}
& \hat{u}_{0}(\xi) \geqslant \hat{w}_{\lambda_{0}}(\xi) \quad \xi \in(0,1), \\
& \left\{\hat{u}_{0}^{\prime}(\xi)+\frac{f(\xi)}{\hat{u}_{0}(\xi)}\right\}^{\prime} \leqslant 0 \quad \xi \in(0,1) .
\end{aligned}
$$

Then we have

$$
\begin{aligned}
& \hat{u}(\xi, \tau) \geqslant \hat{w}_{\lambda_{0}}(\xi) \quad(\xi, \tau) \in(0,1) \times[0, \infty), \\
& \hat{u}(\xi, \tau) \leqslant 0 \quad(\xi, \tau) \in(0,1) \times[0, \infty), \\
& \lim _{\tau \rightarrow \infty} \sup _{0<\xi<1}\left|\hat{u}(\xi, \tau)-\hat{w}_{\lambda_{0}}(\xi)\right|=0 .
\end{aligned}
$$

All the conclusions (7.3) (7.9) of Theorem 7.1 are valid replacing $\lambda$ by $\lambda_{0}$. Especially we have

$$
\begin{array}{r}
(\operatorname{sgn} x)\left\{u\left(x+u^{-1}(\xi, \tau), \tau\right)-w_{\lambda_{0}}\left(x+w_{\lambda_{0}}{ }^{-1}(\xi)\right)\right\} \searrow 0 \\
\text { as } \tau \nearrow+\infty .
\end{array}
$$

This Theorem follows at once from Lemmas 9.1, 9.2 and Theorem 7.1. Note that Theorem 7.1 gives 
ReMark to TheOREM 9.1.

$$
\left\{a_{0}^{\prime}(\xi)+\frac{f(\xi)}{a_{0}(\xi)}\right\}^{\prime} \geqslant 0 \quad \xi \in(0,1)
$$

gives

$$
\hat{u}_{0}(\xi) \leqslant \hat{w}_{\lambda_{0}}(\xi) \quad \xi \in(0,1)
$$

Theorem 9.2. Let us fix any $\lambda$ satisfying $\lambda \geqslant \lambda_{0}$. Suppose that the solutions $u_{k}(x, t)(k=1,2)$ of $(1)$ have the initial functions $u_{k 0}(x)$ which belong to the class $N$. Suppose that $\hat{a}_{k 0}(\xi) \in C^{1}[0,1]$. Suppose that we have

$$
\begin{aligned}
& \hat{a}_{k 0}{ }^{\prime}(0)=\sigma_{-}(\lambda)=\lambda-\sqrt{\lambda^{2}-\lambda_{0}^{2}}, \\
& (-1)^{k}\left\{\hat{a}_{k 0}{ }^{\prime}(1)-\tau_{-}(\lambda)>\right\} 0, \quad \tau_{-}(\lambda)=\lambda-\sqrt{\lambda^{2}-f^{\prime}(1)}, \\
& (-1)^{k}\left\{\hat{a}_{k 0}{ }^{\prime}(\xi)+\frac{f(\xi)}{\hat{u}_{k 0}(\xi)}\right\}^{\prime} \geqslant 0 \quad \xi \in(0,1) .
\end{aligned}
$$

Then we have

$$
\begin{aligned}
& (-1)^{k} \hat{u}_{k}(\xi, \tau) \geqslant 0 \quad(\xi, \tau) \in(0,1) \times[0, \infty), \\
& (-1)^{k}\left\{\hat{w}_{\lambda}(\xi)-\hat{u}_{k 0}(\xi)\right\}>0 \quad \xi \in(0,1), \\
& \lim _{\tau \rightarrow \infty} \sup _{0<\xi<1}\left|\hat{u}_{k}(\xi, \tau)-\hat{w}_{\lambda}(\xi)\right|=0 .
\end{aligned}
$$

All the conclusions $(7.3) \sim(7.9)$ of Theorem 7.1 are valid replacing $u(x, t)$ by $u_{k}(x, t)$. Especially we have

$$
(-1)^{k^{-1}}(\operatorname{sgn} x)\left\{u_{k}\left(x+u_{k}^{-1}(\xi, \tau), \tau\right)-w_{\lambda}\left(x+w_{\lambda}{ }^{-1}(\xi)\right)\right\} \searrow 0
$$

as $\tau \searrow+\infty$.

Proof. We prove the case of $k=1$. The case of $k=2$ can be proved similarly. Lemma 9.2 shows (9.44). To prove this theorem it suffices to show (9.45). The remaining parts follow from Theorem 7.1. Suppose that (9.45) is not valid, since $\hat{w}_{\lambda}(1)=\hat{u}_{10}(1)=0, \hat{w}_{\lambda}^{\prime}(1)-a_{10}{ }^{\prime}(1)=\tau_{-}(\lambda)-\hat{u}_{10}{ }^{\prime}(1)>0$, there exists $\xi_{0} \in(0,1)$ such that

$$
\left\{\begin{array}{l}
\hat{u}_{10}\left(\xi_{0}\right)=\hat{w}_{\lambda}\left(\xi_{0}\right), \quad \hat{u}_{10}{ }^{\prime}\left(\xi_{0}\right) \geqslant \hat{w}_{\lambda}{ }^{\prime}\left(\xi_{0}\right), \\
\hat{u}_{10}(\xi)>\hat{w}_{\lambda}(\xi) \quad \xi \in\left(\xi_{0}, 1\right) .
\end{array}\right.
$$

By (9.41) we have

$$
\left.\left\{\hat{u}_{10}{ }^{\prime}(\xi)+\frac{f(\xi)}{\hat{u}_{10}(\xi)}\right\}\right|_{\xi=+0}=2 \lambda .
$$

(9.43), (9.49) and 


$$
\hat{w}_{\lambda}^{\prime}(\xi)+\frac{f(\xi)}{\hat{w}_{\lambda}(\xi)}=2 \lambda \quad \xi \in(0,1)
$$

give

$$
\hat{u}_{10}{ }^{\prime}\left(\xi_{0}\right)+\frac{f\left(\xi_{0}\right)}{\hat{u}_{10}\left(\xi_{0}\right)} \leqslant 2 \lambda=\hat{w}_{\lambda}{ }^{\prime}\left(\xi_{0}\right)+\frac{f\left(\xi_{0}\right)}{\hat{w}_{\lambda}\left(\xi_{0}\right)} .
$$

(9.48) and (9.50) give

$$
a_{10}{ }^{\prime}\left(\xi_{0}\right)+\frac{f\left(\xi_{0}\right)}{a_{10}\left(\xi_{0}\right)}=2 \lambda .
$$

(9.43), (9.49), (9.51) and (9.48) give

$$
\left\{\begin{array}{l}
\hat{a}_{10}{ }^{\prime}(\xi)+\frac{f(\xi)}{\hat{a}_{10}(\xi)}=2 \lambda \quad \xi \in\left(0, \xi_{0}\right), \\
\hat{a}_{10}\left(\xi_{0}\right)=\hat{w}_{\lambda_{0}}\left(\xi_{0}\right) .
\end{array}\right.
$$

By (21) and (9.52) we have

$$
\hat{u}_{10}(\xi)=\hat{w}_{\lambda}(\xi) \quad \xi \in\left[0, \xi_{0}\right) .
$$

(9.48) and (9.54) show

$$
a_{10}(\xi) \geqslant \hat{w}_{\lambda}(\xi) \quad \xi \in(0,1) .
$$

(9.44) and (9.55) give

$$
\hat{u}_{10}(\xi) \geqslant a_{1}(\xi, \tau) \geqslant \hat{w}_{\lambda}(\xi) \quad(\xi, \tau) \in(0,1) \times[0, \infty) .
$$

(9.54) and (9.56) give

$$
\hat{u}_{10}(\xi)=\hat{u}_{1}(\xi, \tau)=\hat{w}_{\lambda}(\xi) \quad(\xi, \tau) \in\left[0, \xi_{0}\right] \times[0, \infty) .
$$

Since we have the relations:

$$
\begin{aligned}
& x=\int_{\xi_{0}}^{u_{1}\left(x+u_{1}-{ }^{-1}\left(\xi_{0}, \tau\right), \tau\right)} \frac{d \eta}{a_{1}(\eta, \tau)}, \\
& x=\int_{\xi_{0}}^{w_{\lambda}\left(x+w_{\lambda}-{ }^{-1}\left(\xi_{0}\right)\right)} \frac{d \eta}{\hat{w}_{\lambda}(\eta)} .
\end{aligned}
$$

Then it follows

$$
\begin{array}{ll}
u_{1}\left(x+u_{1}{ }^{-1}\left(\xi_{0}, \tau\right), \tau\right)=w_{\lambda}\left(x+w_{\lambda}{ }^{-1}\left(\xi_{0}\right)\right) & x \leqslant 0, \\
u_{1}\left(x+u_{1}{ }^{-1}\left(\xi_{0}, \tau\right), \tau\right) \geqslant w_{\lambda}\left(x+w_{\lambda}{ }^{-1}\left(\xi_{0}\right)\right) & x \geqslant 0 .
\end{array}
$$

Differentiating (9.60) with respect to $\tau$ we get

$$
\left(u_{1}^{-1}\right) \cdot\left(\xi_{0}, \tau\right)=-\frac{\dot{u}_{1}\left(x+u_{1}^{-1}\left(\xi_{0}, \tau\right), \tau\right)}{u_{1}^{\prime}\left(x+u_{1}^{-1}\left(\xi_{0}, \tau\right), \tau\right)} \quad x \leqslant 0 .
$$


Since $\dot{u}_{1}=u_{1}^{\prime \prime}+f\left(u_{1}\right), w_{\lambda}{ }^{\prime \prime}+f\left(w_{\lambda}\right)=2 \lambda w_{\lambda}{ }^{\prime}$, it follows from (9.60) and (9.62) that

$$
\left(u_{1}^{-1}\right) \cdot\left(\xi_{0}, \tau\right)=-2 \lambda \text {. }
$$

This implies

$$
u_{1}^{-1}\left(\xi_{0}, \tau\right)=-2 \lambda \tau+u_{10}^{-1}\left(\xi_{0}\right) .
$$

Let us fix $\nu>0$ sufficiently large so that $F(\xi, \nu)=f(\xi)+\nu \xi$ is monotone increasing with respect to $\xi$ in $(0,1)$. Put

$$
\begin{aligned}
& h(x, t)=e^{\left(\lambda^{2}+v\right) t-\lambda x}\left\{u_{1}\left(x-2 \lambda t+u_{10}^{-1}\left(\xi_{0}\right), t\right)-\right. \\
& \left.-w_{\lambda}\left(x+w_{\lambda}^{-1}\left(\xi_{0}\right)\right)\right\}, \\
& h_{0}(x)=e^{-\lambda x}\left\{u_{10}\left(x+u_{10}^{-1}\left(\xi_{0}\right)\right)-w_{\lambda}\left(x+w_{\lambda}^{-1}\left(\xi_{0}\right)\right)\right\}, \\
& k(x, t)=e^{\left(\lambda^{2}+\nu\right) t-\lambda x}\left\{F\left(u_{1}\left(x-2 \lambda t+u_{10}^{-1}\left(\xi_{0}\right), t\right) ; \nu\right)-\right. \\
& \left.-F\left(w_{\lambda}\left(x+w_{\lambda}^{-1}\left(\xi_{0}\right)\right) ; \nu\right)\right\} .
\end{aligned}
$$

It is easy to see the relations:

$$
\begin{cases}L h=k(x, t) \geqslant 0 & (x, t) \in(0, \infty) \times(0, \infty), \\ h(0, t)=0 & t \in(0, \infty), \\ h(x, 0)=h_{0}(x)>0 & x \in(0, \infty) .\end{cases}
$$

So we have

$$
\begin{aligned}
& h(x, t)=\int_{0}^{\infty}\{H(x-y, t)-H(x+y, t)\} h_{0}(y) d y+ \\
& +\int_{0}^{t} \int_{0}^{\infty}\{H(x-y, t-s)-H(x+y, t-s)\} k(y, s) d y d s .
\end{aligned}
$$

Differentiating with respect to $x$ and putting $x=0$, we get

$$
\begin{aligned}
& h^{\prime}(0, t)=\int_{0}^{\infty} \frac{y}{t} H(y, t) h_{0}(y) d y+ \\
& +\int_{0}^{t} \int_{0}^{\infty} \frac{y}{t-s} H(y, t-s) k(y, s) d y d s .
\end{aligned}
$$

This shows

$$
h^{\prime}(0, t)>0 \quad t \in(0, \infty) .
$$

On the other hand $(9.60)$ gives

$$
h^{\prime}(0, t)=0 \quad t \in(0, \infty) .
$$

This is a contradiction. This proves (9.45) and completes the proof of Theorem

9.2. The following two theorems answer the question how to show the existence 
of such functions as $u_{k 0}(x)(k=1,2)$ in Theorem 9.2. Consider the equation

$$
\hat{w}^{\prime}(\xi)+\frac{f(\xi)}{\hat{w}(\xi)}=2 \lambda+g^{\prime}(\xi) \quad \xi \in(0,1),
$$

giving a smooth function $g(\xi)$ which satisfies $g(0)=g^{\prime}(0)=0$.

Theorem 9.3. Let us fix any $\lambda$ satisfying $\lambda>\lambda_{0}$. Suppose that

$$
\left\{\begin{array}{l}
g^{\prime}(1)<0, \\
g^{\prime \prime}(\xi) \leqslant 0, \quad 2 \lambda+g^{\prime}(\xi)>2 \lambda_{0} \quad \xi \in(0,1) .
\end{array}\right.
$$

Then there exists $w(x) \in N$ for which $\hat{w}(\xi)$ satisfies (9.73) and

$$
\begin{aligned}
& \hat{w}^{\prime}(0)=\sigma_{-}(\lambda), \quad \tau_{-}\left(\lambda_{0}\right)<\hat{w}^{\prime}(1)<\tau_{-}(\lambda), \\
& \hat{w}_{\lambda}(\xi)<\hat{w}(\xi)<\hat{w}_{\lambda_{0}}(\xi) \quad \xi \in(0,1) .
\end{aligned}
$$

Theorem 9.4. Let us fix any $\lambda$ satisfying $\lambda \geqslant \lambda_{0}$. Suppose that

$$
g^{\prime}(1)>0, \quad g^{\prime \prime}(\xi) \geqslant 0 \quad \xi \in(0,1) .
$$

Then there exists $w(x) \in N$ for which $\hat{w}(\xi)$ satisfies (9.73) and

$$
\begin{aligned}
& \hat{w}^{\prime}(0)=\sigma_{-}(\lambda), \quad \hat{w}^{\prime}(1)>\tau_{-}(\lambda), \\
& \hat{w}(\xi)<\hat{w}_{\lambda}(\xi) \quad \xi \in(0,1) .
\end{aligned}
$$

Theorem 9.3 and 9.4 follow at once from Theorems 1.2, 3.1 and 9.2.

Theorem 9.5. Let us fix any $\lambda$ satisfying $\lambda \geqslant \lambda_{0}$. Suppose that $u_{k}(x, t)$ and $u_{k 0}(x)(k=1,2)$ are the functions stated in Theorem 9.2. Suppose that the solution $v(x, t)$ of (1) has the initial function $v_{0}(x) \in M$. If

$$
\hat{u}_{10}(\xi) \geqslant \hat{v}_{0}(\xi) \geqslant \hat{u}_{20}(\xi) \quad \xi \in(0,1)
$$

then we have

$$
\begin{aligned}
& \hat{u}_{1}(\xi, \tau) \geqslant \hat{v}(\xi, \tau) \geqslant \hat{u}_{2}(\xi, \tau) \quad(\xi, \tau) \in(0,1) \times[0, \infty), \\
& \lim _{\tau \rightarrow \infty} \sup _{0<\xi<1}\left|\hat{v}(\xi, \tau)-\hat{w}_{\lambda}(\xi)\right|=0 .
\end{aligned}
$$

All the conclusions (7.3) (7.9) of Theorem 7.1 are valid replacing $u(x, t)$ by $v(x, t)$.

Proof. Replacing $\hat{u}$ and $h$ by $\hat{v}$ and $\hat{u}_{k}$ in (29) we have

$$
\left\{\begin{array}{l}
{\left[\hat{L}_{\hat{v}}-\left\{f^{\prime}(\xi)+\left(\hat{v}+\hat{u}_{k}\right) \hat{u}_{k}{ }^{\prime \prime}\right\}\right]\left(\hat{u}_{k}-\hat{v}\right)=0,} \\
\left.(-1)^{k-1}\left(\hat{u}_{k}-\hat{v}\right)\right|_{\tau=0} \geqslant 0
\end{array}\right.
$$

Since

$$
0<\hat{\imath}(\xi, \tau) \leqslant p(\xi)=c \xi(1-\xi) \quad(\xi, \tau) \in(0,1) \times[0, \infty)
$$




$$
\hat{w}_{\lambda_{1}}(\xi) \leqslant \hat{u}_{2}(\xi, \tau) \leqslant \hat{w}_{\lambda}(\xi) \leqslant \hat{u}_{1}(\xi, \tau) \quad(\xi, \tau) \in(0,1) \times[0, \infty)
$$

for some $c>0$ and $\lambda_{1} \geqslant \lambda_{0}$, it follows

$$
0<\hat{\vartheta}(\xi, \tau) \leqslant C \hat{u}_{k}(\xi, \tau) \quad(\xi, \tau) \in(0,1) \times[0, \infty), \quad k=1,2 .
$$

for some $C>0$. Lemma 9.1 gives

$$
\left|\hat{u}_{k}(\xi, \tau) \hat{a}_{k}{ }^{\prime \prime}(\xi, \tau)\right| \leqslant e^{A \tau+B} \quad(\xi, \tau) \in(0,1) \times[0, \infty)
$$

for some $A \geqslant 0$ and $B \geqslant 0$. By (9.86) and (9.87) we have

$$
\left|f(\xi)+\left(\hat{v}+\hat{u}_{k}\right) \hat{u}_{k}{ }^{\prime \prime}\right| \leqslant e^{A^{\tau+B}} \quad(\xi, \tau) \in(0,1) \times[0, \infty) \quad k=1,2
$$

for some $A \geqslant 0$ and $B \geqslant 0$. This assures the applicability of the comparison theorem to (9.83). Thus we have

$$
(-1)^{k-1}\left\{\hat{u}_{k}(\xi, \tau)-\hat{v}(\xi, \tau)\right\} \geqslant 0 \quad(\xi, \tau) \in(0,1) \times[0, \infty) \quad k=1,2 .
$$

This proves (9.81). The remaining parts of the proof of Theorem 9.5 follow from Theorem 9.2 and 7.1.

\section{An example}

To illustrate the meaning of the results obtained in Section 9 we consider the special example:

$$
\left\{\begin{array}{l}
{\left[\frac{\partial}{\partial t}-\left(\frac{\partial}{\partial x}\right)^{2} u=\lambda_{0}^{2} u\left(1-u^{n}\right), 0 \leqslant u \leqslant 1 \quad(x, t) \in R^{1} \times(0, \infty),\right.} \\
u(x, 0)=u_{0}(x) \quad x \in R^{1} .
\end{array}\right.
$$

Here $\lambda_{0}>0$ and $n=1,2,3, \cdots$. The case of $n=1,(10.1)$ is the combined diffusion and logistic equation which appears in the theories of population dynamics, branching Markov processes and so on. ([4], [5]) The case of $n=2,(10.1)$ is the time dependent Ginzburg-Landau equation which appears in the theory of super conductivity. ([6], [7]) Consider the one parameter family of the initial functions in the class $N$.

$$
u_{0}(x)=\left\{1+\left(2^{n / 2}-1\right) e^{-(n / 2) \sigma}\right\}^{-2 / n} \quad x \in R^{1}, \sigma>0 .
$$

The question is what happens for the solution $u(x, t)$ of $(10.1)$. The answer is as follows

(i) If $\sigma \geqslant \lambda_{0}$, then we have

$$
(\operatorname{sgn} x)\left\{u\left(x+u^{-1}(\xi, \tau), \tau\right)-w_{\lambda_{0}}\left(x+w_{\lambda_{0}}{ }^{-1}(\xi)\right)\right\} \searrow 0 \quad \text { as } \tau \nearrow+\infty .
$$

(ii) If $\lambda_{0}>\sigma=\lambda-\sqrt{\lambda^{2}-\lambda_{0}^{2}}>\sigma_{1}=\left(\frac{n}{2}+1\right)^{-1 / 2} \lambda_{0}$, then we have 


$$
(\operatorname{sgn} x)\left\{u\left(x+u^{-1}(\xi, \tau), \tau\right)-w_{\lambda}\left(x+w_{\lambda}^{-1}(\xi)\right)\right\} \searrow 0 \quad \text { as } \tau \nearrow+\infty .
$$

(iii) If $\sigma=\sigma_{1}=\lambda_{1}-\sqrt{\lambda_{1}^{2}-\lambda_{0}^{2}}$, then we have

$$
\begin{aligned}
& u(x, t)=\left[1+\left(2^{n / 2}-1\right) \exp \left\{-\frac{n}{2} \sigma_{1}\left(x+2 \lambda_{1} t\right)\right\}\right]^{-2 / n} . \\
& \left(2 \lambda_{1}=\left\{\left(\frac{n}{2}+1\right)^{1 / 2}+\left(\frac{n}{2}+1\right)^{-1 / 2}\right\} \lambda_{0}\right)
\end{aligned}
$$

(iv) If $\sigma_{1}>\sigma=\lambda-\sqrt{\lambda^{2}-\lambda_{0}^{2}}>0$, then we have

$$
(\operatorname{sgn} x)\left\{u\left(x+u^{-1}(\xi, \tau), \tau\right)-w_{\lambda}\left(x+w_{\lambda}{ }^{-1}(\xi)\right)\right\} \nearrow 0 \quad \text { as } \tau \nearrow+\infty .
$$

In all the cases the convergence occurs uniformly with respect to $x \in R^{1}$ and uniformly with respect to $\xi$ in every closed subinterval of $(0,1)$. The proof of the above results is based on the following facts:

$$
\begin{aligned}
& \hat{u}_{0}(\xi)=\sigma \xi(1-\xi)^{n / 2} \quad \xi \in(0,1), \\
& \hat{u}_{0}^{\prime}(\xi)+\frac{f(\xi)}{\hat{u}_{0}(\xi)}=\frac{\lambda_{0}^{2}}{\sigma}+\sigma+\left\{\frac{\lambda_{0}^{2}}{\sigma}-\left(\frac{n}{2}+1\right) \sigma\right\} \xi^{n / 2} \quad \xi \in(0,1), \\
& \lambda_{0} \xi\left(1-\xi^{n / 2}\right)>\hat{w}_{\lambda_{0}}(\xi) \quad \xi \in(0,1) .
\end{aligned}
$$

Acknowledgement:

The author wishes to thank Prof. M. Yamaguti for bringing KPP's work to his attention, and Prof. N. Ikeda and Dr. K. Hayakawa for their valuable discussions.

\section{Osaka City University}

\section{References}

[1] A. Kolmogoroff, I. Petrovsky and N. Piscounoff: Étude de l'equation de la diffusion avec croissance de la quantité de matière et son application à un problème biologique, Bull. Univ. d'État à Moscou, Ser. Internat. Sect. A, I (1937), 1-25.

[2] V. Lakshmikantham and S. Leela: Differential and Integral Inequalities, Vol. II, Academic Press, 1969.

[3] E.A. Coddington and N. Levinson: Theory of Ordinary Differential Equations, McGraw-Hill, 1955.

[4] R. Fisher: The Genetical Theory of Natural Selection, Oxford University Press, 1930.

[5] S. Watanabe: Limit theorem for a class of branching processes, Markov Processes and Potential Theory, Edited by J. Chover, John Wiley and Sons, 1967, 205-232.

[6] E. Abrahams and T. Tsuneto: Time variation of the Ginzburg-Landau order parameter, Phys. Rev. 152 (1966), 416-432.

[7] D.E. McCumber and B.I. Halperin: Time scale of intrinsic resistive fluctuations in thin superconducting wires, Phys. Rev. B 1 (1970), 1054-1070. 